\title{
Produktinnovationen und Produktmodifikationen in der Versicherungswirtschaft zwischen 2006 und 2017
}

\author{
Thomas Köhne $\mathbb{D} \cdot$ Inna Melashenko
}

Online publiziert: 10. Januar 2020

(C) Der/die Autor(en) 2020

Zusammenfassung Die vorliegende empirische Untersuchung erfasst die Produktinnovationen und Produktmodifikationen im deutschen Versicherungsmarkt für Privatkunden; sie schließt an eine frühere Erhebung von 1996 bis 2005 an und untersucht die weitere Entwicklung der Produktneuerungen zwischen 2006 und 2017. Im theoretischen Teil werden begriffliche Grundlagen geklärt, die förderlichen und hinderlichen Rahmenbedingungen der Neuproduktentwicklung reflektiert sowie Arbeitshypothesen abgeleitet. Im empirischen Teil werden im Privatkundengeschäft 1457 Produktneuerungen identifiziert, davon sind 79 Produktinnovationen und 1378 Produktmodifikationen. Damit hat die Anzahl der Produktneuerungen im Vergleich zum früheren Zeitraum deutlich zugenommen und sich von durchschnittlich 65 auf 121 Produkte pro Jahr fast verdoppelt. Nach wie vor gibt es mit fast $95 \%$ der Produktneuerungen erheblich mehr Produktmodifikationen als Produktinnovationen. Sowohl in der Lebens-, als auch in der Krankenversicherung wird nach wie vor ein Großteil der Produktneuerungen durch politisch-rechtliche Anstöße verursacht. Klassische Lebensversicherungsprodukte mit Garantiezins werden zunehmend von Alternativen ohne Garantien ersetzt. In der Schaden-/Unfallversicherung sind Produktneuerungen demgegenüber oft Technologie- oder Risiko-induziert. Mithin hat das Produktneuerungsgebaren der Versicherer im deutschen Privatkundenmarkt in den zurückliegenden 12 Jahren - wenngleich auf moderatem Niveau - offensichtlich zugenommen, und das in allen Versicherungszweiggruppen.

Schlüsselwörter Produktentwicklung · Produktneuerung · Produktinnovation · Produktmodifikation · Privatkunden · Lebensversicherung · Krankenversicherung • Schaden-/Unfallversicherung

T. Köhne $(\bowtie)$

Campus Lichtenberg, Hochschule für Wirtschaft und Recht Berlin, Haus 5,

Alt-Friedrichsfelde 60, 10315 Berlin, Deutschland

E-Mail: thomas.koehne@hwr-berlin.de 


\title{
Product innovations and product modifications in the German insurance industry in the years between 2006 and 2017
}

\begin{abstract}
This empirical analysis comprises product innovations and product modifications in the German insurance industry for the private customer segment; it takes up a previous analysis concerning the years between 1996 and 2005 and examines the progress of the development of new products for the years between 2006 and 2017. In the theoretical part of the paper, definitions are given, positive as well as restraining framework conditions are reflected and working hypothesis are deduced. In the empirical part of the paper, 1457 new products are identified in the private customer segment; 79 of those are product innovations and 1378 of those are product modifications. Thus, the number of new products has increased considerably compared to the previous period and the average number of new products per year has almost doubled from 65 to 121. Similar to the previous study, almost $95 \%$ of new products are product modifications which means that there are still much more product modifications than product innovations. In the life insurance as well as in health insurance, a large proportion of new products are still caused by political and regulatory impetus. Traditional participating life insurance products with year-toyear guarantees are increasingly replaced by life insurance products with alternative or without any guarantees. In comparison, in the property and casualty insurance, new products are resulting many times from technological developments or from newly emerging risks. Hence, over the last 12 years the activities for developing new products in the German insurance industry in the private customer segment have increased (but still at a moderate level) in every insurance line.
\end{abstract}

Keywords Product development - Product innovation · Product modification · Private customer $\cdot$ Life insurance $\cdot$ Health insurance $\cdot$ Property and casualty insurance

\section{Einleitung}

Mit der Deregulierung im Jahr 1994 haben sich die produktgestalterischen Spielräume der Versicherer deutlich verbessert. ${ }^{1}$ Daher ging eine empirische Untersuchung im Jahr 2007 der Frage nach den Auswirkungen auf das Produktneuerungsgebaren der Versicherer nach. Sie wies erstmals quantitativ nach, dass im Zeitraum nach der Deregulierung (konkret: zwischen 1996 und 2005) branchenweit sowohl Produktinnovationen (mit 2,4 pro Jahr) als auch Produktmodifikationen (mit 63 pro Jahr) nur eine überschaubare Anzahl angenommen haben und stufte mithin die Bedeutung von Produktneuerungen als relativ gering ein. Und $27 \%$ aller deutschen Versicherer hatten in den damals untersuchten zehn Jahren (1996-2005) nur ein neues Produkt entwickelt. Ferner wurde gezeigt, dass die Bedeutung in diesen zehn Jahren keine signifikante Veränderung erfahren hatte. Zudem wurde geschlussfolgert, dass die Deregulierung für sich allein genommen somit nur begrenzt Impulse für vermehrte

\footnotetext{
1 Vgl. Farny (1995, S. 80).
} 
Produktneuerungen in der deutschen Versicherungsbranche geliefert hatte. Begründet wurde das - neben den empirischen Befunden - damit, dass Produktneuerungen in der Versicherungswirtschaft vor dem Hintergrund eines hohen unternehmerischen Risikos bei gleichzeitig fehlenden Monopolrenten aus wertorientierter Sicht eine hohe Bürde auferlegt wird und sich folglich die Rolle von Produktneuerungen in Abhängigkeit von ihrem Wert- und Kundennutzenbeitrag bestimmt. ${ }^{2}$

Weil nach der damaligen Untersuchung keine entsprechenden Folgeuntersuchungen durchgeführt wurden, soll die vorliegende empirische Untersuchung diese Forschungslücke schließen, an die damalige Erhebung anknüpfen und entsprechend den Zeitraum von 2006 bis 2017 erfassen. Dabei soll sie erstens die weitere Entwicklung hinsichtlich der Produktneuerungen im deutschen Versicherungsmarkt ermitteln und untersuchen, ob sich diese im Vergleich zum Zeitraum von 1996 bis 2005 verändert hat, insbesondere ob die Anzahl an Produktneuerungen nun doch noch wie nach der Deregulierung erwartet spürbar gestiegen oder ob sie vielleicht sogar zurückgegangen ist. Zweitens soll überprüft werden, inwieweit die Erkenntnisse von damals bestätigt werden können. Drittens sollen neue Erkenntnisse gewonnen werden: Denn im hier betrachteten Zeitraum haben sich einige rechtliche, Finanzmarkt-bezogene und technologische Rahmenbedingungen - teils fundamental - geändert. Zu nennen sind diesbezüglich beispielsweise das umfassende Regulierungsregime Solvency II, das Unisex-Urteil des EuGH, der Provisionsdeckel in der Krankenversicherung, das Lebensversicherungsreformgesetz, das Anleger seit 2007 herausfordernde, EU-weit historisch tiefe Zinsniveau sowie technologische Entwicklungen auf den Gebieten Web-Technologie, Künstliche Intelligenz, Big Data oder Blockchain.

Die Abhandlung ist wie folgt aufgebaut: Im folgenden Abschnitt werden zunächst kurz die theoretischen Überlegungen von damals reflektiert und weiterentwickelt. Auf dieser Basis werden verschiedene Hypothesen aufgestellt. Der dritte Abschnitt ist der empirischen Untersuchung gewidmet: Zunächst wird das Vorgehen beschrieben, daraufhin werden die Ergebnisse dargestellt und mit den Ergebnissen der Studie von 2007 verglichen sowie die Hypothesen überprüft. Der abschließende Abschnitt dient den Schlussfolgerungen, der kritischen Würdigung und dem Blick auf die künftige Entwicklung.

\section{Produktneuerungen in der Versicherungswirtschaft aus theoretischer Sicht}

\subsection{Begriffliche Unterscheidung zwischen Produktinnovation und Produktmodifikation}

Die Definitionen einer Produktinnovation und einer Produktmodifikation werden von Köhne/Kopp aus 2007 übernommen: Danach ist ,die Produktinnovation (..) ein Versicherungsprodukt inklusive seiner prozessualen Ausprägungen im Dienstleistungsprozess - beispielsweise im Fall der Schadenleistung -, das im Hinblick auf die Befriedigung des Bedürfnisses nach finanzieller Risikovorsorge oder damit

\footnotetext{
2 Vgl. Köhne und Kopp (2007).
} 
in funktionsorientiertem Zusammenhang stehender Bedürfnisse bislang auf dem relevanten Markt noch nicht angeboten worden ist und insofern eine Marktneuheit darstellt. “3 Als Produktmodifikation werden demgegenüber ,,alle anderen Veränderungen an bestehenden Versicherungsprodukten, die ,nur' Anpassungen, Ergänzungen und Flexibilisierungen des versicherten Bereichs bzw. des Kernproduktes darstellen“4, definiert. Die Produktmodifikation umfasst damit sowohl Variationen als auch Differenzierungen bestehender Versicherungsprodukte: Eine Produktvariation ist die Weiterentwicklung einzelner Komponenten, um das Produkt für den Versicherungsnehmer attraktiver zu machen, z.B. indem Kernprodukte durch Nebenrisiken erweitert werden. Eine Produktdifferenzierung weitet bestehende Deckungskonzepte z. B. auf neue Zielgruppen und Regionalmärkte aus und flexibilisiert diese. $^{5}$

Zwei Gründe sprechen für die Übernahme der Definitionen: Erstens wird die Vergleichbarkeit der Ergebnisse der damaligen Untersuchung mit der aktuellen Untersuchung nur gewährleistet, wenn die zu unterscheidenden Kategorien der Innovation und der Modifikation gleich definiert werden. Zweitens erscheinen beide Definitionen und die damit verbundene begriffliche Abgrenzung nach wie vor sinnvoll und zweckdienlich. ${ }^{6}$

\subsection{Rolle von Produktneuerungen aus Unternehmens-, Vermittler- und Kundensicht}

Auch die grundsätzlichen Aussagen zur Rolle von Produktneuerungen von 2007 gelten nach wie vor: ${ }^{7}$

Aus Unternehmenssicht sind Produktinnovationen im Kontext ziel- und strategieorientierter Überlegungen zu beurteilen. Produktinnovationsziele gehen im Versicherungsunternehmen mit den formalen Unternehmenszielen einher und tragen in unterschiedlicher Intensität zur Erreichung dieser Ziele bei: ${ }^{8}$ Hinsichtlich des Umsatzstrebens können Produktinnovationen in Form von kundenorientierten Problemlösungen die Stornowahrscheinlichkeit reduzieren und die Wahrscheinlichkeit des Folgekaufs erhöhen, zudem erlauben sie ab dem Zeitpunkt der Markteinführung die Abschöpfung von Monopolrenditen. Produktmodifikationen unterstützen zumindest das Neugeschäft (Wachstumsziel). Insofern können beide damit mengenseitig eine Umsatzsteigerung induzieren. Kostenseitig resultieren aus jeglichen Produktneuerungen steigende Betriebskosten in Folge der erfolgten Produktentwicklungsarbeiten, notwendigen Marketings sowie der Anpassung der Bestandssysteme. Allerdings könnten hier moderne, modulare Produktbausteine sowie infolge der Digitalisierung

\footnotetext{
3 Köhne und Kopp (2007, S. 233).

4 Ebenda.

5 Vgl. ebenda, S. $232 \mathrm{f}$.

6 Bezüglich der Herleitung und der entsprechenden Quellenangaben wird daher auf Köhne und Kopp (2007, S. 230-233) verwiesen. Vgl. hierzu und nachfolgend auch Köhne (2016, S. 278).

7 Siehe hierzu und zur entsprechenden Literatur: Köhne und Kopp (2007, S. 233-238).

8 Vgl. hierzu und für die nachfolgenden Absätze Köhne und Kopp (2007, S. 234f.); Farny (2011, S. 332-353).
} 
flexiblere IT-Systeme Produktentwicklungs- und IT-Kosten reduzieren. ${ }^{9}$ Bei Produktinnovationen fallen zudem in aller Regel höhere Sicherheitskapitalkosten an, weil das versicherungstechnische Risiko aufgrund geringerer Erfahrungswerte in der Schätzung der Schadenverteilung höher ist. ${ }^{10}$ Das Gewinnziel wird mithin letztlich nur dann unterstützt, wenn neue Produkte wirklich zu mehr Umsatz führen und/ oder mit niedrigeren Betriebskosten einhergehen. Im Sinne wertorientierter Steuerung des Versicherungsunternehmens wird die Rolle der Produktneuerungen daher davon abhängen, inwiefern sie dem Innovator nachhaltig Wert schaffen.

Wenngleich das Ziel der Kundenorientierung (Bedarfsdeckung) Produktneuerungen geradezu erfordert, können dem vor allem bei Produktinnovationen das Gewinnbzw. Wertschaffungsziel entgegenstehen: Fehlende Sonderschutzrechte und oftmals zu geringes Kundeninteresse gefährden die Monopolrente von Produktinnovatoren (so genannten First Movern), zugleich sind damit hohe unternehmerische Risiken versicherungstechnischer Art verbunden.

Produktneuerungen determinieren die ökonomischen Ziele überdies auf indirektem Wege: Sie unterstützen die Erreichung kundenbezogener, psychologischer Ziele wie die Verbesserung des Bekanntheitsgrades oder des Images des Versicherungsunternehmens. Ein positives Unternehmensimage beeinflusst die Einstellung der Kunden und erleichtert dem Vermittler die Ansprache der (potenziellen) Kunden.

Ungeachtet der genannten ökonomischen Ziele genießt bei Versicherungsunternehmen die eigene Existenzsicherheit oberste Priorität; das resultiert aus der Natur des Geschäfts und aus umfangreichen rechtlichen Vorgaben zum Schutz der Versicherungsnehmeransprüche. Somit bildet das Sicherheitsziel eine Nebenbedingung für die übrigen Unternehmensziele. Einerseits determiniert die Erreichung dieses Nebenziels die Beurteilung der Produktqualität durch Kunden und Vermittler, konkret in Form von Ratingergebnissen. Andererseits kann es das Produktneuerungsgebaren von Versicherern hemmen, weil es naturgemäß deren Risikoaversion fördert. ${ }^{11}$

Unter der Bedingung der nachhaltigen Existenzsicherung ist aus Unternehmenssicht mithin das primäre Ziel von Produktneuerungen, durch Unterstützung der übergeordneten, formalen Unternehmensziele die Wettbewerbssituation des Unternehmens zu verbessern. Entsprechend spielen Produktinnovationen und Produktmodifikationen nach wie vor eine wichtige Rolle bei der Umsetzung von Unternehmensstrategien, beispielsweise im Zuge der Geschäftsstrategie (Kosten-, Differenzierungs- oder Nischenstrategie). Bei der Nischenstrategie ist besonders darauf hinzuweisen, dass diese eine bedarfsgerechte Leistungsgestaltung im Hinblick auf spezielle Marktnischen, beispielsweise Zielgruppen, verlangt und sich vor allem Produktmodifikationen zur Umsetzung von derartigen Zielgruppenprodukten eignen, da oftmals nur Deckungsvariationen notwendig sind.

\footnotetext{
9 Vgl. vbw Vereinigung der Bayerischen Wirtschaft e. V. (2017, S. 21 und 63).

10 Vgl. Schmeiser et al. (2006, S. 49f.).

11 Dieses Phänomen scheint bei InsurTechs weniger ausgeprägt zu sein, da die mit start-ups oft einhergehende ,Trial-and-Error“-Philosophie für eine geringere Risikoaversion spricht. Allerdings bleibt abzuwarten, inwiefern dies künftig mit aufsichtsrechtlichen Zielen wie Sicherheit und Nachhaltigkeit im Einklang steht.
} 
Die Vermittlersicht auf Produktneuerungen differenziert sich von jener der Versicherer. Das Zielsystem der Vermittler ist dadurch geprägt, Einkommensziele zu erreichen, die ihrerseits stark an Umsatz- und Provisionsziele gekoppelt sind, sowie die mit der Erreichung der Einkommensziele verbundenen Kosten so gering wie möglich zu halten. Die Kosten resultieren in erster Linie aus dem Zeitaufwand für die Vor- und Nachbereitung von Kundenbesuchen, der eigenen Qualifizierung sowie Verwaltungskosten. ${ }^{12}$

Vor diesem Hintergrund sind Produktinnovationen für Vermittler dann attraktiv, wenn sie sich leicht verkaufen lassen, weil der Kundenbedarf sehr groß ist (und die Konkurrenzangebote fehlen) und wenn die damit verbundenen Monopolrenten $\mathrm{zu}$ besonders hohen Provisionen führen. In diesem Fall werden mit Produktinnovationen verbundene Zusatzkosten sowie ein zusätzlicher Qualifizierungs- und Beratungsaufwand überkompensiert. Produktmodifikationen sind für Vermittler sogar noch attraktiver, da sie einen vergleichsweise geringeren Schulungs- und Beratungsaufwand generieren und trotzdem marketingseitig dem Vermittler Argumente für Kundenbesuche offerieren. Allerdings erlauben sie im Allgemeinen keine großen Prämienerhöhungen und insofern wenig Provisionszugeständnisse. Zudem steigen mit zunehmender Zahl an Produktvarianten infolge häufiger Produktmodifikationen die Kosten der Komplexität. Aus Vermittlersicht lässt sich grundsätzlich festhalten, dass Produktneuerungen von diesen gewünscht werden und der Stärkung der Kundenbindung und des Neugeschäfts dienen, soweit sie hinsichtlich Anzahl und Taktung überschaubar bleiben. ${ }^{13}$

Schließlich ist mit Blick auf die Kundensicht zu konstatieren, dass mit der durch die Digitalisierung nachhaltig gestiegenen Transparenz eine Verschiebung des „Machtverhältnisses“ vom Versicherer als Produzenten zum Versicherungsnehmer als Konsumenten stattfindet. Das Kommunikationsverhalten verändert sich dadurch. ${ }^{14}$ Das gilt jedoch weniger für das tatsächliche Nachfrageverhalten: Die Finanzbildung und das Wissen um Risiken und entsprechende Absicherungsmöglichkeiten in der Bevölkerung sind insgesamt gering, das Gleiche gilt für die Bereitschaft, sich überhaupt mit Versicherungsthemen zu beschäftigen. Am ehesten dürfte dies noch für den kleinteiligen situativen Versicherungsschutz gelten, der mit den neuen Zugangswegen in Zukunft deutlich einfacher ,on demand“ abgeschlossen werden kann, wie etwa einer kurzfristigen Reise- oder Unfallversicherung oder einer tageweise hinzugebuchten Kfz-Versicherung. Fraglich ist jedoch, inwieweit neue Kommunikationsmöglichkeiten Menschen dazu bringen werden, sich stärker mit Versicherungsfragen zu beschäftigen, ihren Versicherungsschutz häufiger an einen veränderten Bedarf oder eine veränderte Marktsituation anzupassen und dabei Produktneuerungen überhaupt wahrzunehmen. ${ }^{15}$ Insofern gilt (auch heute immer noch), dass Versicherungskunden im Privatkundengeschäft aufgrund ihres weitgehend fehlenden Interesses wenig dazu beitragen, die theoretisch wichtige Rolle und mit der

\footnotetext{
12 Vgl. Lach (1995, S. 115 und 140-142).

13 Vgl. Köhne (2016, S. 270f.); mit Bezug auf Assistanceleistungen Müller-Reichart et al. (2019, S. 36f.).

14 Vgl. Lohse und Will (2019, S. 10f.).

15 Vgl. Theis und Wiener (2018, S. 19).
} 
Deregulierung erwartete zunehmende Bedeutung von Produktneuerungen nachfrageseitig zu fördern. ${ }^{16}$

\subsection{Förderliche und hinderliche Rahmenbedingungen für Produktneuerungen}

In der Versicherungsbranche liefern äußere Rahmenbedingungen einen wichtigen Impuls für die Entwicklung neuer Produkte. ${ }^{17}$ Deswegen soll nun geklärt werden, inwiefern die 2006 identifizierten förderlichen und hinderlichen Rahmenbedingungen heute immer noch gelten und inwiefern die im Einleitungsabschnitt angedeuteten, aktuelleren Entwicklungen im regulatorischen Umfeld, auf den Kapitalmärkten oder im technologischen Bereich die Entstehung von Produktneuerungen beeinflussen.

Als förderliche Rahmenbedingungen für Produktneuerungen wurden damals politisch-rechtliche Anstöße (Deregulierung der Bedingungs- und Tarifgestaltung, Anpassungen von Rechtsnormen, Rechtsprechung), die demografische und technologische Entwicklung sowie verschiedene Eigenschaften des Versicherungsproduktes selbst herausgestellt. ${ }^{18}$ Hinzu gekommen ist die seit der Finanzkrise von 2007 veränderte Kapitalmarktsituation, die Produktneuerungen verlangt hat.

Deregulierung der Bedingungs- und Tarifgestaltung: Die damit verbundene Freiheit in der Produktgestaltung besteht immer noch.

Anpassungen von Rechtsnormen durch neue Gesetze oder Gesetztesanpassungen sowie neue rechtliche Rahmenbedingungen durch Rechtsprechung: Nach wie vor gilt, dass Gesetzgebung und Rechtsprechung Produktneuerungen geradezu erzwingen, von den verbraucherpolitisch motivierten Regulierungen jedoch vor allem die Personenversicherungszweige wie die Lebens- und die Krankenversicherung betroffen sind. ${ }^{19}$ Neue Gesetze oder Rechtsurteile wie der 2012 gesetzlich vorgeschriebene Provisionsdeckel in der Krankenversicherung ${ }^{20}$, das Lebensversicherungsreformgesetz von $2014^{21}$ oder das sogenannte Unisex-Urteil des EuGH von $2011^{22}$ beeinflussen die Kalkulation in den Personenversicherungszweigen. Es ist davon auszugehen, dass sie entsprechende Produktanpassungen in den jeweiligen Jahren nach sich gezogen haben. Somit sollten auch zwischen 2006 und 2017 politisch-rechtliche Anstöße zu umfangreichen Produktneuerungen in der Personenversicherung geführt haben.

Demografische Entwicklungen: Sie beeinflussen direkt die Kalkulation in den Personenversicherungszweigen. Das geschieht fortlaufend und verändert nicht zwingend die Produktlandschaft. Allerdings dürfte die grundlegende Verschiebung der Altersstruktur weg von jungen hin zu alten Menschen neue Produktkonzepte ins-

\footnotetext{
16 Vgl. Köhne (2016, S. 278).

17 Vgl. Swiss Re (Hrsg.) (2011, S. 11-23).

18 Vgl. hierzu und nachfolgend Köhne und Kopp (2007, S. 238f.).

19 Vgl. Köhne (2016, S. 265-267).

20 Umgesetzt in Art. 22 des Gesetzes zur Novellierung des Finanzanlagenvermittler- und Vermögensanlagenrechts vom 6. Dez. 2011.

21 Gesetz zur Absicherung stabiler und fairer Leistungen für Lebensversicherte (Lebensversicherungsreformgesetz - LVRG) vom 1. Aug. 2014.

22 Urteil des Europäischen Gerichtshofes vom 1. März 2011 (Rechtssache C-236/09).
} 
besondere in der Rentenversicherung fördern. ${ }^{23}$ Ebenso dürften damit verbundene gesellschaftliche Trends wie zunehmender Convenience- und gegebenenfalls auch Pflegebedarf im Alter Service- und Assistanceprodukte beflügeln. ${ }^{24}$ Aber auch gesellschaftliche Trends im Segment junger Menschen, wie die Entwicklungen der Generation Y und Z, beeinflussen die Produktgestaltung; beispielsweise bieten gerade InsurTechs neuartige Lösungen an, die dem Kommunikations- und CommunityVerhalten (z. B. Teilen statt Besitzen) dieser Zielgruppen entsprechen und veränderte Versicherungslösungen bedingen. ${ }^{25}$

Technologische Entwicklungen: Deren Bedeutung für die Entstehung von Produktneuerungen erklärten Köhne und Kopp (2007) damit, dass bei der Entwicklung neuer Technologien in den Jahren zwischen 1997 und 2007 ein regelrechter Quantensprung vollzogen worden sei, nahezu unbegrenzte Kapazitäten für die Speicherung von elektronischen Daten vorhanden seien, das Internet im privaten und wirtschaftlichen Kontext eine fast selbstverständliche Plattform zum Austausch von multimedialen Informationen und Nachrichten aller Art geworden sei und diese Basisentwicklungen zu Veränderungen sowohl bei der Neugestaltung als auch bei der Verwendung von Versicherungsprodukten geführt hätten. Neuartige Produktentwicklungswerkzeuge würden ein verkürztes ,time to market“, eine erhöhte Produktflexibilität und eine zentrale Abbildbarkeit von Produktwissen versprechen, und am Point of Service könnten elektronische Medien neue Produkte besser im Hinblick auf ihre Problemlösungswirkung visualisieren und Entscheidungen zum Underwriting durch die Anbindung der Vermittler an die IT des Versicherers rascher getroffen werden. ${ }^{26}$ Im Vergleich zu heute standen diese Entwicklungen damals jedoch noch „in den Kinderschuhen“. Seitdem haben Versicherer sich weiter industrialisiert, die Digitalisierung ist deutlich vorangeschritten und die Möglichkeiten von Web-Anwendungen haben zahlreiche, innovative Start ups entstehen lassen, die sich zudem im Einsatz von Künstlicher Intelligenz (z. B. im Underwriting) und von Big Data (z. B. im Bereich der Kalkulation) üben. Die zahlreichen FinTechs und InsurTechs haben selbst neue Produkte entwickelt oder Versicherer dazu motiviert, dies zu tun. ${ }^{27}$ Technologische Entwicklungen dürften also auch in den vergangenen Jahren und heute förderlich für Produktneuerungen sein. ${ }^{28}$

Eigenschaften des Versicherungsproduktes: Als förderlich für die Entwicklung von Produktneuerungen wurden damals die Immaterialität und die Digitalisierbarkeit sowie damit die kostengünstige Multiplizierbarkeit, der geringe Finanzierungsbedarf aufgrund des Vorfinanzierungseffekts durch die Prämienzahlungen der Versicherungsnehmer sowie die Ergänzungsfähigkeit um zahlreiche Serviceleistungen

\footnotetext{
23 Vgl. De Ridder et al. (2015, S. 79-85); Wichert (2016).

24 Vgl. Müller-Reichart und Geist (2015, S. 255f.); Lohse und Will (2019, S. 4).

25 Vgl. MSR Insights/W\&W Digital GmbH/etventure GmbH (Hrsg.) (2016).

26 Vgl. Köhne und Kopp (2007, S. 239).

27 Vgl. Wiener und Theis (2017); Oliver Wyman/Policen Direkt (Hrsg.) (2017, S. 13, 16-17); BaFin (Hrsg.) (2018, S. 95-134).

28 So auch bei Eling und Lehmann (2018, S. 369).
} 
genannt. ${ }^{29}$ Diese Produkteigenschaften sind grundlegender Natur und haben sich nicht verändert.

Die Finanzkrise 2007 hat nicht nur eine zunehmende Regulierung bewirkt, sondern zugleich den Kapitalmarkt nachhaltig beeinflusst: Seitdem ist das Zinsniveau historisch niedrig. Im Zusammenspiel mit der zwar niedrigen, aber dennoch positiven Inflationsrate ergibt sich für Sparer eine reale Negativverzinsung. ${ }^{30}$ Diese Situation hat in den letzten Jahren vor allem die Lebensversicherung unter starken Druck gebracht. In Verbindung mit der erwähnten Regulierung trägt das dazu bei, dass einige Versicherer ihr Neugeschäft einstellen (interner Run off) ${ }^{31}$ und einige überdies Bestände verkaufen oder übertragen (externer Run off). ${ }^{32}$ Bezogen auf das Produktneuerungsgebaren, sollte diese Zinssituation dazu führen, dass insbesondere die Lebensversicherer zunehmend neue Produkte auf den Markt bringen, die weniger Garantien und dafür mehr Rendite versprechen. ${ }^{33}$

Als hinderliche Rahmenbedingungen für Produktneuerungen wurden demgegenüber damals politisch-rechtliche Einschränkungen (jederzeitige politisch motivierte Veränderbarkeit des Altersvorsorge- und Gesundheitssystems, aufsichtsrechtliche Vorschriften hinsichtlich Produkt- und Preisgestaltung, fehlender Patentschutz), bestimmte Eigenschaften des Versicherungsproduktes selbst, die Branchenkultur sowie das geringe Kundeninteresse an Versicherungsprodukten herausgestellt. ${ }^{34}$ Hinzugekommen ist 2016 das neue Aufsichtsregime Solvency II.

Politisch-rechtliche Einschränkungen: Die damals aufgeführten Aspekte betrafen zunächst einmal die jederzeit möglichen, politisch motivierten und potenziell drastischen Veränderungen des Altersvorsorge- und Gesundheitssystems, welche vor allem in der Personenversicherung die Rahmenbedingungen weniger berechenbar gemacht haben. Dieser Umstand ist heute nach wie vor gegeben. Zudem wurden diverse einschränkende aufsichtsrechtliche Rahmenbedingungen angeführt wie Spartentrennungsgebot, Verbot versicherungsfremder Geschäfte, Grundsatz der Gleichbehandlung der Versicherungsnehmer in der Lebens-, Kranken- und Unfallversicherung mit Prämienrückgewähr bei Prämien und Leistungen und damit Verzicht auf klassische Preisdifferenzierungsmaßnahmen sowie Provisionsabgabeverbot. Auch diese Regelungen gelten immer noch. Das Provisionsabgabeverbot wurde sogar 2018

\footnotetext{
${ }^{29}$ Im Einzelnen wird auf die damaligen Aussagen und Quellen verwiesen: Siehe Köhne und Kopp (2007, S. 239).

30 Vgl. Brühl und Walz (2015, S. 2). Dort werden deutsche Bundesanleihen kurzer und mittlerer Laufzeit zugrunde gelegt, die am langen Ende z. B. bei 10-jährigen Bundesanleihen eine nur sehr geringe positive Verzinsung mit sich bringen.

${ }^{31}$ So hat bspw. die ERGO verkündet, dass sie bezüglich aller ,gängigen Formen der Lebens- und Rentenversicherung (..) das Neugeschäft im Wesentlichen eingestellt" hat (ERGO Lebensversicherung AG (Hrsg.) 2019, S. 4).

${ }^{32}$ So haben 2018 die (irische) Axa ihren „Twinstar“-Bestand und die Generali 89,9\% ihres Leben-Bestandes an die Viridium Gruppe veräußert (vgl. Surminski 2018, S. 503); Generali Deutschland AG (Hrsg.) (2019).

33 Vgl. Bierth et al. (2018, S. 134). Zum Zusammenhang zwischen Garantie und Rendite siehe Ruß et al. (2018, S. 45-48).

${ }^{34}$ Vgl. hierzu und nachfolgend Köhne und Kopp (2007, S. 240f.).
} 
erst explizit in das VAG aufgenommen. ${ }^{35}$ Dafür ist eine Regelung im damaligen $\S 49$ VVG zur Begrenzung des Schadenersatzes eines Versicherers auf Geldleistungen weggefallen. Schließlich wurden die fehlenden Exklusivrechte angeführt. Auch diese Argumentation gilt immer noch: Weil Versicherungsprodukte nicht vom Patent-, Urheber- oder Wettbewerbsrecht geschützt werden, sind Deckungskonzepte leicht zu imitieren und nachhaltige Wettbewerbsvorsprünge durch Schaffung neuer Versicherungsdeckungen kaum möglich. ${ }^{36}$

Das sehr umfassende Regulierungsregime Solvency II ist zum 1. Januar 2016 in Kraft getreten. Dieses verfolgt im Rahmen der Harmonisierung des Aufsichtsrechts im Europäischen Wirtschaftsraum das Ziel, umfassenden Verbraucherschutz zu gewährleisten und das Insolvenzrisiko von Versicherern zu vermeiden. Basierend auf dem Drei-Säulen-Ansatz, schreibt Solvency II eine risikobasierte Bestimmung des Solvabilitätskapitals und des Mindestkapitals vor und formuliert Anforderungen an die Eigenmittelausstattung der Versicherer. Einerseits wirkt es dabei hinsichtlich Produktneuerungen möglicherweise förderlich, weil es zu Produktmodifikationen bzw. sogar zu Produktinnovationen führt, indem Versicherer bestehende Produkte ,risikoärmer machen“. Beispielsweise haben viele Versicherer sogenannte Neue KlassikTarife in der Lebensversicherung entwickelt und auf den Markt gebracht, die weniger Kapitalerhaltungsgarantien als traditionelle Klassik-Tarife mit herkömmlichem Garantiezins erfordern und damit im Sinne von Solvency II Eigenmittel-schonender sind. ${ }^{37}$ Zugleich wirkt Solvency II jedoch hinderlich, weil vor allem Produktinnovationen oftmals mit einem höheren versicherungstechnischen Risiko einhergehen und damit mehr Eigenmittel erfordern. ${ }^{38}$ Langfristig - also nach Umstellung auf „risikoärmere“ Produkte - dürfte der negative Effekt für Produktinnovationen überwiegen.

Eigenschaften des Versicherungsproduktes: Die leichte Kopierbarkeit, das versicherungstechnische Risiko bei der Produktkalkulation (insbesondere bei Produktinnovationen), die besondere Bestandsproblematik durch die Kombination von andauernder Reifephase und geringer Produktsterblichkeit, die oft langfristigen Versicherungsverträge sowie der daraus resultierende Nachlaufeffekt ,alter Produkte“ über Jahrzehnte, der die produktpolitischen Wirkungen auf die Unternehmensziele verringert, führten zu folgendem damaligen Fazit: „Es besteht ein Spannungsfeld zwischen der tendenziell langfristigen Ausrichtung von Versicherungsprodukten und der kurzfristigen Reaktion auf Markterfordernisse. “39 Gilt das 2019 immer noch? Im Grunde gelten die gleichen Produktbedingungen, insbesondere in der Lebens- und in der Krankenversicherung. In den Schadenversicherungszweigen gibt es jedoch zumindest etwas veränderte Rahmenbedingungen in Bezug auf die Langfristigkeit der

\footnotetext{
35 Das ist über Art. 2 Zif. 7 des Gesetzes zur Umsetzung der Richtlinie (EU) 2016/97 des Europäischen Parlaments und des Rates vom 20. Januar 2016 über Versicherungsvertrieb und zur Änderung weiterer Gesetze vom 20. Juli 2017 geschehen.

36 Vgl. Gal (2019, S. 102-111).

37 Vgl. Reuß et al. (2016); Deutsche Aktuarvereinigung e.V. (Hrsg.) (2017, S. 12f.); Klotzki (2018, S. 144).

38 Vgl. Schmeiser et al. (2006, S. 49f.); Bierth et al. (2018, S. 138).

39 Köhne und Kopp (2007, S. 241).
} 
Verträge: Seit der VVG-Reform 2007 sind die unkündbaren Vertragslaufzeiten auf drei Jahre reduziert worden, die Versicherer können auch kurzfristigere Laufzeiten vereinbaren, und wettbewerbsbedingt geschieht dies auch. ${ }^{40}$ So gibt es insbesondere InsurTechs, die tägliche oder monatliche Kündigungsmöglichkeiten anbieten. ${ }^{41}$ Auch wenn das besagte Spannungsfeld durch kürzere Vertragslaufzeiten abnimmt und insbesondere InsurTechs auf eine kurzfristige Marktreaktion abzielen, bleibt es dennoch prinzipiell bestehen. Denn vielen Risiken - vor allem in der Alters-, Gesundheitsund Unfallvorsorge - sind Versicherungsnehmer langfristig ausgesetzt, und dort sind langfristige Ansparvorgänge gefragt und zentrales Prinzip der Versicherungsidee. ${ }^{42}$

Branchenkultur: Das Argument einer im Branchenvergleich geringeren Erfahrung im Management von Produktinnovationen gilt 25 Jahre nach der Produktderegulierung nicht mehr. Auch die 2006 konstatierte, noch überwiegend konservative Einstellung und eher skeptische Haltung gegenüber Neuem hat sich nicht zuletzt durch den Blick auf und die Zusammenarbeit mit InsurTechs verändert; die Gründung von „Digital Labs“ durch immer mehr Versicherer ist nur ein Zeichen dessen. ${ }^{43}$ Allenfalls die branchenimmanente Sicherheitsorientierung, die heute durch Solvency II und die gestiegene Bedeutung von Compliance-Regelungen sogar noch verstärkt wird, ist der Branche nach wie vor zu attestieren - sie ist jedoch vor allem Folge der zunehmenden Regulierung. Die Branchenkultur an sich scheint somit kein relevantes Hindernis von Produktneuerungen mehr darzustellen.

Geringes Kundeninteresse an Versicherungsprodukten: Das überwiegend schwach ausgeprägte Interesse von Versicherungskunden an Versicherungsprodukten und das in der Regel geringe Versicherungs-Know how sind über die Jahre konstant geblieben. Bis heute haben daran auch die neuen Kommunikationsmöglichkeiten durch soziale Netzwerke oder über Apps (noch) nichts geändert - im Gegenteil: Die sogenannte „Generation Y“ ist in ihrer Lebensplanung noch zurückhaltender gegenüber Versicherungen als vorherige gleichaltrige Kohorten. ${ }^{44}$ Mithin stellt das fehlende Kundeninteresse derzeit immer noch eines der entscheidendsten Hindernisse für Produktneuerungen dar.

\subsection{Hypothesen zur Entwicklung von Produktneuerungen zwischen 2006 und 2017}

Auch im Zeitraum von 2006 bis 2017 dürften typische Rahmenbedingungen der Produktentwicklung wie die Abhängigkeit von Gesetzen und Rechtsprechung insbesondere in der Lebens- und Krankenversicherung und von demografisch-gesell-

\footnotetext{
${ }^{40}$ Genauer gesagt beträgt die unkündbare Höchstfrist anstatt zuvor 5 Jahren nur noch drei Jahre. Längere Vertragslaufzeiten sind möglich, aber der Versicherungsnehmer hat danach ein gesetzliches Kündigungsrecht nach Ablauf von jeweils 12 Monaten ( $\$ 11 \mathrm{IV}$ VVG).

${ }^{41}$ Dazu gehören beispielsweise Fri:Day (monatlich kündbare Kfz-Versicherung) (FRI:DAY 2019), Getsafe (täglich kündbare Haftpflichtversicherung) (Getsafe 2019), MAXCARE (täglich kündbare Zahnzusatzversicherung der Deutschen Familienversicherung) (MAXCARE 2019); oder Hepster (täglich kündbare Reiseunfallversicherung) (Hepster 2019).

42 Vgl. Farny (2011, S. 54f.).

43 Vgl. Wiener und Theis (2017, S. 3).

44 Vgl. Gaedeke (2016, S. 89f.).
} 
schaftlichen Entwicklungen sowie die Eigenschaften des Versicherungsproduktes Produktneuerungen induzieren. Technologische Entwicklungen dürften noch mehr Dynamik entfachen als vor 2006. Die Wirkung komplexer Aufsichtsregime wie Solvency II ist ambivalent; kurzfristig - also im Untersuchungszeitraum - dürften sie Produktmodifikationen und sogar Produktinnovationen (Stichwort: Neue Klassik) befördern, langfristig - also außerhalb des Untersuchungszeitraums - zumindest Produktinnovationen eher hemmen.

Somit ist zu erwarten, dass die Anzahl an Produktmodifikationen wegen rechtlicher Anforderungen (z.B. infolge Unisex-Urteil, LVRG, Solvency II) und damit verbundener Anpassungsnotwendigkeiten zu den entsprechenden Zeitpunkten zunimmt. Ebenso dürfte das andauernde niedrige Zinsniveau Produktneuerungen nach sich ziehen. Zudem entfachen neue technologische Möglichkeiten in Kombination mit veränderten Anforderungen der Kunden an Kommunikation und Services eine beständige fördernde Wirkung auf Produktneuerungen. Die Digitalisierung aller Lebensbereiche erfordert die interne Umgestaltung der Versicherungsunternehmen und bringt neue Kundenansprüche mit sich: Studien bestätigen, dass Kunden eine schnelle Kommunikation mit dem Versicherer über Online-Plattformen wünschen und digitale, einfache und verständliche Produkte erwarten, die schnell abrufbar sind und auf den individuellen Bedarf zugeschnitten sind. ${ }^{45}$ Diesbezüglich bieten sich Versicherungsprodukte mit kurzer Laufzeit und Produkte mit häufigerem Kundenkontakt (z.B. in der Betreuung oder im Schadenfall) an, also eher Schadenversicherungen. Das Gleiche gilt für die Nachfrage nach Serviceleistungen.

Daraus lassen sich nunmehr folgende Arbeitshypothesen ableiten, die in der empirischen Untersuchung überprüft werden sollen:

H 1 Im Zeitraum zwischen 2006 und 2017 nimmt die Anzahl an Produktneuerungen im Vergleich zum Zeitraum zwischen 1996 und 2005 insgesamt zu.

H 2 Die zunehmende Anzahl an Produktneuerungen geht nur auf die Produktmodifikationen zurück; die Anzahl an Produktinnovationen bleibt indessen gleich.

H 3 Nach wie vor gibt es erheblich mehr Produktmodifikationen (damals waren das über $95 \%$ der Produktneuerungen ${ }^{46}$ ) als Produktinnovationen.

H 4 In der Lebens- und in der Krankenversicherung wird ein Großteil der Produktneuerungen durch politisch-rechtliche Anstöße verursacht.

H 5 Klassische Lebensversicherungsprodukte mit Garantiezins werden von Alternativen ohne Garantien (vor allem fondsgebundene Lebensversicherungen) ersetzt.

H 6 In der Schadenversicherung geht ein großer Teil der Produktneuerungen auf die Digitalisierung und mithin technologische Entwicklungen zurück.

\footnotetext{
45 Vgl. Kotalakidis et al. (2016, S. 4-7).
}

46 Vgl. Köhne und Kopp (2007, S. 245). 
H 7 In der Schadenversicherung geht ein großer Teil der Produktneuerungen auf Service- und Assistanceleistungen zurück.

\section{Empirische Untersuchung von Produktneuerungen im Zeitraum zwischen 2006 und 2017}

\subsection{Datensatz und methodisches Vorgehen}

Um einen Überblick über die Anzahl der Produktneuerungen zu erhalten, wurden im Sinne eines empirisch-quantitativen Vorgehens alle identifizierten Produktneuerungen zwischen 2006 und 2017 in einer Datenbank erfasst. Die dabei erhobenen Daten sind aus ausgewählten Versicherungsfachzeitschriften generiert worden, in denen Produktneuerungen regelmäßig publiziert werden, namentlich aus den Versicherungsfachzeitschriften AssCompact und Versicherungsmagazin. Diese sind besonders auf die Zielgruppe der Versicherungsvermittler ausgerichtet und stellen somit für Versicherungsunternehmen eine geeignete Plattform dar, um für neue Versicherungsprodukte zu werben und ihre Vorteile aufzuzeigen. Beide Fachzeitschriften sind als weitgehend vollständige Quellen eingeschätzt worden, weil aus Marketinggesichtspunkten davon auszugehen ist, dass Versicherer dort über alle relevanten Neuerungen informieren (abgesehen vom Direktvertrieb). ${ }^{47}$ Deswegen wurden die Kriterien der Vollständigkeit (zumindest in Bezug auf das Privatkundengeschäft) und Reliabilität als erfüllt angesehen. Diese Vorgehensweise hatte sich darüber hinaus in der damaligen Studie bewährt. ${ }^{48}$

Gegenstand der Untersuchung sind alle im deutschen Versicherungsmarkt zwischen 2006 und 2017 neu eingeführten oder als neu bezeichneten Versicherungsprodukte gewesen. Dabei sind für alle 288 im Betrachtungszeitraum erschienenen Ausgaben der beiden Zeitschriften im Hinblick auf die Produktneuerungen, die im Versicherungsmagazin in der Rubrik „Neue Produkte“ (bzw. ab dem Jahr 2014 „Neue Versicherungsprodukte“) bzw. in der Zeitschrift AssCompact unter der Überschrift „News“ publiziert worden sind, in Anlehnung an Köhne/Kopp ${ }^{49}$ folgende Variablen erfasst worden: ${ }^{50}$

\footnotetext{
${ }^{47}$ Beide Fachzeitschriften sind jedoch vor allem auf Vermittler im Privatkundengeschäft (und im Gewerbekundensegment) ausgerichtet. Somit ist davon auszugehen, dass dort die meisten Industrieversicherungs-, aber auch bestimmte Gewerbeversicherungsprodukte nicht aufgegriffen werden und sich diese folglich mit der hier gewählten Methodik nicht vollständig erfassen lassen. Dennoch sind die identifizierten Gewerbe-/Industrieversicherungsprodukte erfasst worden, um sie von den Privatkundenprodukten abzugrenzen und zumindest einen ersten groben Einblick in das Unternehmenssegment zu erhalten. Für tiefere und detailliertere Erkenntnisse ist jedoch eine eigenständige empirische Erhebung notwendig.

${ }^{48}$ Vgl. Köhne und Kopp (2007, S. 243f.). Die dort zusätzlich berücksichtigte Zeitschrift Versicherungswirtschaft wurde jedoch außer Acht gelassen, da die Publikationen in dieser kaum Ergänzungen zu den bereits im Versicherungsmagazin und AssCompact veröffentlichten Neuerungen lieferten.

49 Vgl. Köhne und Kopp (2007, S. 243 f.).

${ }^{50}$ Die Daten sind in der Bibliothek des Deutschen Vereins für Versicherungswissenschaft in Berlin erhoben, in dem Programm Excel erfasst sowie anschließend mithilfe der Datenbanksoftware SPSS Statistics ausgewertet worden.
} 
- Name des Versicherungsunternehmens: Resultieren Produkte aus Kooperationen, z.B. zwischen Maklern und Versicherungsunternehmen, ist zwecks der Vermeidung von Doppelzählungen gleicher Produkte stets der Risikoträger erfasst worden. Ist der Risikoträger nicht ersichtlich gewesen, ist der Vermittler erfasst worden.

- Ehemaliger Name: Sollte es Unternehmen geben, die aufgrund von Fusionen in anderen Unternehmen aufgegangen sind oder umfirmiert wurden, ist der neue Unternehmensname und darüber hinaus der ehemalige Name erfasst worden, um die Querverbindung zu der jeweiligen Quelle herzustellen.

- Versicherungsgruppe: Um tiefergehende Aussagen auf Konzernebene treffen zu können, sind die Einzelunternehmen, über die Eingrenzung bei Köhne/Kopp hinaus, den ihnen übergeordneten Konzernen bzw. Gruppen zugeordnet worden.

- Versicherungszweiggruppe: Die Produktneuerungen sind den Versicherungszweiggruppen Leben (L), Kranken (K) und Schaden/Unfall (S) zugeordnet worden, um die Entwicklungen innerhalb der Versicherungszweiggruppen und zwischen beiden Untersuchungen vergleichen zu können.

- Versicherungszweig: Name des jeweiligen Versicherungszweigs.

- Zeitpunkt: Monat und Jahr der Berichterstattung.

- Name des Produkts: Durch die Berücksichtigung des Produktnamens ist gewährleistet worden, dass ein und dasselbe Produkt nur einmal erfasst wird. Bei fehlender Angabe ist der Name entweder auf der Internetseite des jeweiligen Versicherers recherchiert oder durch die allgemeine Produktbezeichnung ersetzt worden.

- Kundensegment: Es ist eine Unterteilung in die Kundensegmente Privatkunden sowie Gewerbe-/Industriekunden vorgenommen worden.

- Zielgruppe: Falls die Zielgruppe benannt worden ist oder auf diese aus der Beschreibung des Produktes geschlossen werden konnte, ist sie explizit erfasst worden.

- Neue Nutzenkomponente: Zu den Produktinnovationen ist der Inhalt der Neuerung, ausgehend von den Informationen im jeweiligen Artikel, aufgegriffen und damit offengelegt worden, aus welchem Grund die Neuerung als Produktinnovation beurteilt wurde.

- Politischer Anstoßpunkt: Politisch-rechtliche Veränderungen sind registriert worden, wenn sie eindeutig die Neuerung ausgelöst haben. Zugrunde gelegt worden sind im Wesentlichen folgende Anstoßpunkte: GKV-Modernisierungsgesetz (wirksam zum 01.01.2004) mit den daraus erwachsenden neuen Tarifen in der Zusatz- und Pflegeversicherung, Reform der gesetzlichen Rentenversicherung 2005, Alterseinkünftegesetz (wirksam zum 01.01.2005), GKV-Wettbewerbsstärkungsgesetz (wirksam zum 01.04.2007), Gesetz zur Förderung der Vermögensbildung der Arbeitnehmer 2009, Gesetz zur Angemessenheit der Vorstandsvergütung (wirksam zum 31.07.2009), GKV-Finanzierungsgesetz (wirksam zum 01.01.2011), Unisex-Urteil von 2012, Lebensversicherungsreformgesetz (wirksam zum 01.08.2014).

- Quelle: Hier ist das Journal erfasst worden, in dem die Neuerung publiziert wurde. Wurde eine Produktneuerung häufiger als nur in einem Journal bzw. einer Ausgabe erwähnt, ist sie lediglich einfach registriert worden. 
Die zeitliche Eingrenzung des Untersuchungsmaterials auf den Zeitraum zwischen 2006 und 2017 ist bewusst erfolgt, um Überschneidungen mit der Untersuchung von Köhne/Kopp zu vermeiden und explizit zu untersuchen, inwiefern sich der Untersuchungszeitraum von der damals untersuchten Zeitspanne zwischen 1996 und 2005 unterscheidet.

Die Produkte sind entsprechend der festgelegten Kriterien den Kategorien „Produktinnovation“ oder „Produktmodifikation“ zugeordnet worden. Im Rahmen der empirisch-quantitativen Forschung ist die Rolle und Bedeutung von Produktinnovationen und -modifikationen anhand des Kriteriums der Häufigkeit operationalisiert und anschließend mithilfe von Verfahren der deskriptiven Statistik analysiert worden, um sie mit den Ergebnissen von Köhne/Kopp vergleichen zu können.

Durch das gewählte Forschungsdesign sollten mithin die Vergleichbarkeit mit früheren Ergebnissen und die Reproduktion der empirischen Erhebung gewährleistet werden.

\subsection{Zuordnung zu Kategorien}

Die Zuordnung zu Produktinnovationen oder Produktmodifikationen ist anhand der Definition aus Abschn. 2.1 erfolgt:

Ausschlaggebend gewesen für die Charakterisierung als Produktinnovation ist dabei vor allem das Kriterium der Neuartigkeit. Es beschreibt, worin die eigentliche Neuerung besteht. Die Artikel wurden dementsprechend mit dem Ziel durchgesehen, neue Zwecke und/oder neue Mittel zur Erfüllung der Zwecke und somit eine neue Zweck-Mittel-Beziehung zu identifizieren. Hierbei wurde anhand der inhaltlichen Beschreibung entschieden, ob das Produkt eine Abwandlung oder Erweiterung bestehender Deckungskonzepte ist oder tatsächlich eine neue Nutzenkomponente besitzt.

Der Kategorie „Produktinnovation“ zugeordnet wurden Produkte bei Bildung einer neuen Versicherungssparte und eines neuen versicherten Bereichs durch neue versicherte Personen, Sachen oder Interessen sowie bei neuen versicherten Schäden. Hinweise in den Artikeln, dass ein Produkt den Innovationspreis erhalten hat oder eine neue Komponente besitzt, sind hierbei kritisch hinterfragt worden, und das Produkt ist lediglich bei erfolgreicher Identifikation der neuen Nutzenkomponente als Innovation kategorisiert worden.

Eine Produktmodifikation umfasst demgegenüber Variationen und Differenzierungen bestehender Versicherungsprodukte hinsichtlich des Umfangs des versicherten Bereichs in Form von Hinzufügungen, Verringerungen oder Variation versicherter Elemente. Entsprechend sind alle Produkte, die als Tariferweiterung oder -verbesserung bezeichnet werden, der Kategorie „Produktmodifikation“ zugeordnet worden. $\mathrm{Da}$ in diesen Fällen häufig nicht ersichtlich ist, inwiefern ein bestehendes Produkt verändert wurde, musste auf die Unterteilung in Variation bzw. Differenzierung verzichtet werden. Alle Produktneuerungen, die auf politisch-rechtlichen Anstoßpunkten basieren, sind den Produktmodifikationen zugeordnet worden, da bei diesen der erste Anbieter nicht die Idee generiert hat, sondern sie lediglich als erster umgesetzt hat. 
Nicht als Produktneuerungen erfasst worden sind kurzfristige und saisonale Sonderaktionen der Versicherer, die ebenfalls in den Versicherungsfachzeitschriften beworben werden. Der Grund dafür ist, dass es sich bei diesen meist um Preisnachlässe und Rabatte handelt, die keine Veränderung des Produktes an sich mitbringen und nach Ablauf der Aktion nicht mehr angeboten werden. Ebenfalls nicht als Produktneuerungen eingestuft wurden Prozessänderungen z.B. in Form von veränderten Antragsverfahren, Onlinevertriebskanälen oder Online-Tarifrechnern, bei denen keine neue Nutzenkomponente identifiziert werden kann. Dasselbe gilt z. B. für die Ausweitung der Fondspaletten für fondsgebundene Versicherungsprodukte, da der Produktkern unverändert bleibt.

Im Privatkundensegment ist die Vorgehensweise von Köhne/Kopp übernommen und die Aufteilung der Versicherungszweige nach Farny ${ }^{51}$ vorgenommen worden. Ebenfalls wurde ergänzend Anlage A zum VAG verwendet. Im Verlauf der Zeit sind jedoch Produkte entstanden, die sich nicht eindeutig den definierten Versicherungszweigen zuordnen lassen oder neue Versicherungszweige begründen; das gilt naturgemäß insbesondere für Produktinnovationen. In solchen Fällen sind Bezeichnungen verwendet worden, die am ehesten auf den Inhalt der Versicherung schließen lassen, so z. B. die Bezeichnung „Hochzeitsversicherung“. Lebensversicherungsprodukte, die sich nicht eindeutig einem Versicherungszweig zuordnen lassen, sind unter „Altersvorsorge“ zusammengefasst worden. Als Hybridprodukte werden solche Lebensversicherungen verstanden, bei denen die zur Leistungserfüllung bestimmten Kapitalanlagen zum Teil in fondsgebundenen Kapitalanlagen und zum Teil im Anlagestock des klassischen Lebensversicherungsgeschäfts gehalten werden. ${ }^{52}$ Ebenfalls sind Variable Annuity-Produkte aufgrund ihrer eigenständigen Charakters separat aufgeführt worden. Zusätzlich berücksichtigt worden sind Produkte der betrieblichen Altersvorsorge.

Zusätzlich ist als Versicherungszweig „Service/Assistance“ aufgenommen worden. Darunter fallen Produkte, die ausschließlich zusätzlichen Service bieten, sowie bestehende Produkte, die um Servicekomponenten ergänzt werden.

\subsection{Ergebnisse der empirischen Untersuchung}

\subsubsection{Gesamtüberblick über die Produktneuerungen in der Versicherungsbranche zwischen 2006 und 2017}

Im Rahmen der empirischen Untersuchung sind für den Zeitraum von 2006 bis 2017 insgesamt 1750 Produktneuerungen erfasst worden, davon 1457 im Privatkundenund 293 im Gewerbe- und Industriekundensegment. Von den 1750 Produktneuerungen sind über beide Kundensegmente hinweg 90 als Produktinnovationen im Sinne der zugrunde gelegten Definition identifiziert worden. Das entspricht etwa fünf Prozent aller Neuproduktideen. Zugleich wurden 1660 Produktmodifikationen erfasst; das sind mehr als 18-mal so viele Modifikationen wie Innovationen (Tab. 1).

51 Vgl. Farny (2011, S. 241-243).
52 Vgl. Gabler Versicherungslexikon (2017, S. 414f.). 
Tab. 1 Anzahl der erfassten Produktneuerungen nach Kundensegmenten

\begin{tabular}{llll}
\hline & Innovationen & Modifikationen & Gesamt \\
\hline Privatkunden & 79 & 1378 & 1457 \\
Gewerbe- und Industriekunden & 11 & 282 & 293 \\
Gesamt & 90 & 1660 & 1750 \\
\hline
\end{tabular}

Tab. 2 Anzahl der Produktneuerungen nach Versicherungszweiggruppen (über alle Kundensegmente)

\begin{tabular}{llll}
\hline & Innovationen & Modifikationen & Gesamt \\
\hline Lebensversicherung & 25 & 511 & 536 \\
Krankenversicherung & 9 & 262 & 271 \\
Schaden-/Unfallversicherung & 56 & 887 & 943 \\
Gesamt & 90 & 1660 & 1750 \\
\hline
\end{tabular}

Tab. 3 Anzahl der Produktneuerungen im Zeitverlauf (über alle Kundensegmente)

\begin{tabular}{llll}
\hline & Innovationen & Modifikationen & Gesamt \\
\hline 2006 & 11 & 152 & 163 \\
2007 & 9 & 146 & 155 \\
2008 & 8 & 142 & 150 \\
2009 & 9 & 196 & 205 \\
2010 & 5 & 139 & 144 \\
2011 & 5 & 111 & 116 \\
2012 & 8 & 95 & 103 \\
2013 & 4 & 132 & 136 \\
2014 & 6 & 131 & 137 \\
2015 & 13 & 132 & 145 \\
2016 & 7 & 148 & 155 \\
2017 & 5 & 136 & 141 \\
Gesamt & 90 & 1660 & 1750 \\
\hline
\end{tabular}

Insgesamt fällt auf, dass mit 54\% aller Neuerungen die meisten Produktneuerungen, sowohl Innovationen als auch Modifikationen, aus der Schaden- und Unfallversicherung stammen (Tab. 2). Im Zeitraum von 1996 bis $2005^{53}$ lag die Lebensversicherung noch vorne.

Im Hinblick auf die Verteilung und Entwicklung der Produktneuerungen im Zeitverlauf (Tab. 3) lässt sich als Befund festhalten, dass die Verteilung über die zwölf Jahre hinweg sowohl bei Innovationen als auch bei Modifikationen relativ gleichmäßig ist. Besonders fällt das Jahr 2009 mit 9 Innovationen und 196 Modifikationen auf. In diesem Jahr sind die meisten Modifikationen erfasst worden, und es ist das einzige Jahr mit über 200 Produktneuerungen. Die meisten Innovationen sind 2015 entstanden; hiervon stammen 7 Innovationen aus der Schaden-/Unfallversicherung. Zusammenhänge zwischen den Produkten sind aber nicht ersichtlich, und man kann keine möglichen externen Gründe für die Produktinnovationen erkennen. Das Jahr

53 Vgl. Köhne und Kopp (2007, S. 249). 
2012 mit 103 Produktneuerungen hat mit Abstand am wenigsten neue Produkte hervorgebracht. Verglichen mit dem Zeitraum von 1996 bis 2005 wäre es aber (gemeinsam mit 2005) ein Spitzenjahr gewesen.

Weitere Befunde beziehen sich auf die Bedeutung politisch-rechtlicher Impulse: Über die Kundensegmente hinweg sind 286 von 1660 Produktmodifikationen auf politisch-rechtliche Veränderungen zurück zu führen. Mit 271 von insgesamt 1378 Produktneuerungen (knapp 20\%) ist ihre Bedeutung im Privatkundensegment deutlich höher als im Gewerbe- und Industriekundensegment. Hier sind lediglich 15 von 282 Neuerungen auf einen politisch-rechtlichen Anstoß zurückzuführen; mithin kann festgestellt werden, dass der politisch-rechtliche Einfluss auf die Produktentwicklung hier sehr gering zu sein scheint.

\subsubsection{Produktneuerungen im Privatkundensegment}

Die Befunde zum gesamten Privatkundensegment umfassen interessante Aussagen zur Anzahl an Produktneuerungen, zum Verhältnis von Produktinnovationen zu Produktmodifikationen sowie zur Bedeutung von Zielgruppenprodukten.

Im Privatkundenbereich hat sich die Anzahl der Produktneuerungen mit 1457 im Vergleich zum Zeitraum von 1996 bis 2005 mit $650^{54}$ mehr als verdoppelt; da allerdings der zweite Untersuchungszeitraum zwei Jahre länger gewesen ist, ist ein Vergleich der durchschnittlichen Jahreswerte aussagekräftiger: Zwischen 2006 und 2017 sind jährlich durchschnittlich 121 Produkte auf den Versicherungsmarkt gebracht worden, zwischen 1996 und 2005 waren es jährlich durchschnittlich 65 Neuerungen. Das bedeutet, dass sich die Anzahl der im Durchschnitt jährlich eingeführten Produkte näherungsweise verdoppelt hat. Hypothese 1 lässt sich somit bestätigen: Im Zeitraum zwischen 2006 und 2017 nimmt die Anzahl an Produktneuerungen im Vergleich zum Zeitraum zwischen 1996 und 2005 insgesamt zu.

Ferner ist das Verhältnis von Innovationen zu Modifikationen von 1:26 im Zeitraum von 1996 bis $2005^{55}$ auf 1:17 im Zeitraum von 2006 bis 2017 gesunken, was aus der leicht überproportionalen Zunahme der Produktinnovationen resultiert. Somit kann Hypothese 2, dass die zunehmende Anzahl an Produktneuerungen auf die Produktmodifikationen zurückgeht, während die Anzahl an Produktinnovationen gleich bleibt, nicht bestätigt werden.

Fast $95 \%$ aller Neuerungen lassen sich den Produktmodifikationen zuordnen. Das bedeutet, dass relativ gesehen der Anteil der Innovationen lediglich um ein Prozent zugenommen hat. Der allgemeine Trend zwischen 1996 und 2005, dass es auf dem Versicherungsmarkt erheblich weniger Innovationen als Modifikationen gibt, hat sich somit fortgesetzt. Damit kann Hypothese 3 ebenfalls bestätigt werden: Nach wie vor gibt es (mit einem Anteil von fast $95 \%$ der Produktneuerungen) erheblich mehr Produktmodifikationen als Produktinnovationen (Tab. 4).

$44 \%$ aller Modifikationen stammen aus dem Schaden- und Unfallbereich, 37\% aus der Lebens- und $19 \%$ aus der Krankenversicherung. Zugleich wurden 79 Pro-

54 Vgl. hier und nachfolgend Köhne und Kopp (2007, S. 245).

55 Vgl. Köhne und Kopp (2007, S. 249). 
Tab. 4 Anzahl der Produktneuerungen im Privatkundensegment nach Versicherungszweiggruppen

\begin{tabular}{llll}
\hline & Innovationen & Modifikationen & Gesamt \\
\hline Lebensversicherung & 25 & 510 & 535 \\
Krankenversicherung & 8 & 260 & 268 \\
Schaden-/Unfallversicherung & 46 & 608 & 654 \\
Gesamt & 79 & 1378 & 1457 \\
\hline
\end{tabular}

Tab. 5 Anzahl der Produktneuerungen mit Zielgruppen-Fokus im Privatkundensegment nach Versicherungszweiggruppen

\begin{tabular}{lll}
\hline & Mit Zielgruppen-Fokus & Ohne Zielgruppen-Fokus \\
\hline Lebensversicherung & 142 & 393 \\
Krankenversicherung & 76 & 192 \\
Schaden-/Unfallversicherung & 183 & 471 \\
Gesamt & 401 & 1056 \\
\hline
\end{tabular}

duktinnovationen identifiziert, die zu 58\% auf Schaden-/Unfall-, zu 32\% auf Lebens- und zu $10 \%$ auf Krankenversicherungsprodukte entfallen (Tab. 4).

Hinzuweisen ist auf die Bedeutung von Zielgruppenprodukten. Sie machen einen Anteil von immerhin $28 \%$ aller Produktneuerungen aus, und das nicht nur - wie man erwarten könnte - in der Schaden-/Unfallversicherung, sondern in ähnlichem Ausmaß auch in der Lebens- und in der Krankenversicherung (Tab. 5). Ferner fällt auf, dass die Bedeutung von Produktneuerungen mit Zielgruppen-Fokus im Vergleich zum Zeitraum zwischen 1996 und 2005 deutlich zugenommen hat; damals waren es nur knapp über zehn Prozent. ${ }^{56}$

Produktneuerungen in der Lebensversicherung Insgesamt stammen im Privatkundensegment $37 \%$ aller identifizierten Produktneuerungen aus dem Lebensversicherungsbereich. Der prozentuale Anteil der Lebensversicherungsprodukte, gemessen an der Gesamtanzahl der Produktneuerungen, hat somit zwar im Vergleich zum Zeitraum zwischen 1996 und 2005 um sechs Prozent abgenommen, macht aber mit 535 Produktneuerungen weiterhin die zweitgrößte Versicherungszweiggruppe im Privatkundensegment aus.

Die stabile Entwicklung und die insgesamt große Anzahl der Produktneuerungen in der Lebensversicherung sind vermutlich auf das lange anhaltende Niedrigzinsumfeld und den damit verbundenen Wandel zurückzuführen. Bislang waren Lebensversicherungsprodukte aufgrund ihrer hohen Garantieverzinsung bei der deutschen Bevölkerung als Finanzprodukte sehr beliebt. ${ }^{57}$ Aufgrund des Niedrigzinsumfeldes und der zunehmenden rechtlichen Vorgaben können Versicherer die klassischen Produkte jedoch nicht mehr im bisherigen Ausmaß anbieten. Damit lassen sich die Bemühungen der Versicherer erklären, durch neue Produkte den Versicherungsnehmern annehmbare Alternativen anbieten zu wollen. ${ }^{58}$

56 Vgl. Köhne und Kopp (2007, S. 252).

57 Vgl. KPMG (Hrsg.) (2018, S. 7).

58 Vgl. Brauns (2016). 
Der Trend hin zu fonds-, zertifikats- und indexgebundenen Versicherungsprodukten, die allesamt Kapitalmärkte ausnutzen, kann anhand der Ergebnisse der empirischen Untersuchung bestätigt werden: Insgesamt sind 217 Produkte identifiziert worden, die alternative Anlageformen in den Vordergrund stellen und einen Teil des Risikos, z. B. bei negativer Kursentwicklung, auf den Kunden übertragen. Das entspricht $41 \%$ aller Produktneuerungen im Lebensversicherungsbereich. Hinzu kommen zusätzlich Produkte der betrieblichen Altersvorsorge, die ebenfalls eine fondsgebundene Rentenversicherung als Grundlage haben. Die Ergebnisse der Untersuchung bestätigen damit die Hypothese 5, dass klassische Lebensversicherungsprodukte mit Garantiezins zunehmend von Alternativen ohne Garantien (vor allem fondsgebundene Lebensversicherungen) abgelöst werden.

Weitere Befunde zur Versicherungszweiggruppe Lebensversicherung betreffen Produkte zur Absicherung biometrischer Risiken und Produkte der betrieblichen Altersvorsorge. Im Untersuchungszeitraum wurden immer mehr neue Produkte zur Absicherung biometrischer Risiken eingeführt. Fasst man Produkte wie die Berufsunfähigkeits-, Dienstunfähigkeits-, Erwerbsunfähigkeits-, Sportunfähigkeitsund Grundfähigkeitsversicherung sowie die Risikolebens-, Dread Disease- und Pflegerentenversicherung zusammen, erhält man 171 Produktneuerungen, wovon 11 Produktinnovationen sind. Die zunehmende Bedeutung der Biometrieprodukte spiegelt sich auch in deren Umsatz wider. ${ }^{59}$ Offensichtlich entwickeln Versicherer vermehrt neue Produkte in diesem Produktbereich, weil zum einen der Bedarf seitens der Kunden vorhanden ist ${ }^{60}$ und zum anderen dieser Produktbereich eine Alternative zum schwächelnden Altersvorsorgebereich bietet.

Darüber hinaus wurden zwischen 2006 und 2017 auch vermehrt Produkte aus dem Bereich der betrieblichen Altersvorsorge eingeführt, so beispielsweise die betriebliche Arbeitsunfähigkeitsversicherung. Dieser Trend kann als Reaktion auf den steigenden betrieblichen Krankenstand und die mit einer steigenden Nachfrage nach betrieblicher Altersvorsorge einhergehenden erhöhten präventiven Bemühungen der Arbeitgeber interpretiert werden, denn zwischen Dezember 2001 und Dezember 2015 ist in Deutschland der Anteil der Betriebsstätten mit einer betrieblichen Altersversorgung von 31 auf $49 \%$, d.h. 18 Prozentpunkte, gestiegen. ${ }^{61}$

Im Vergleich der Versicherungszweiggruppen im Privatkundensegment fällt auf, dass in der Lebensversicherung in absoluten Zahlen mit Abstand die meisten Produktmodifikationen mit politisch-rechtlichem Anstoßpunkt anzutreffen sind (Tab. 6).

Die hohe Anzahl der politisch-rechtlich angeregten Produktmodifikationen lässt sich vor allem auf gesetzliche Änderungen durch das Alterseinkünftegesetz mit Inkrafttreten im Jahr 2005 zurückführen, die einen erheblichen Einfluss auf die eingeführten Varianten der Riester- und Rürüp-Rente hatten. Ebenfalls hat das Gesetz zur Förderung der Vermögensbildung der Arbeitnehmer aus dem Jahr 2009 dazu beigetragen, dass Produkte der betrieblichen Altersvorsorge in abgewandelten Varianten und größerem Umfang auf den Markt gebracht wurden.

\footnotetext{
59 Vgl. KPMG (Hrsg.) (2018, S. 8).

60 Vgl. ebenda., S. 9-12.

61 Vgl. Bundesministerium für Arbeit und Soziales (Hrsg.) (2016, S. 13f.).
} 
Tab. 6 Anzahl Produktmodifikationen mit politisch-rechtlichem Anstoß im Privatkundensegment nach Versicherungszweiggruppen

\begin{tabular}{llll}
\hline & $\begin{array}{l}\text { Anzahl mit pol.- } \\
\text { rechtl. Anstoß }\end{array}$ & $\begin{array}{l}\text { Anzahl ohne pol.- } \\
\text { rechtl. Anstoß }\end{array}$ & Anzahl Gesamt \\
\hline Lebensversicherung & 152 & 358 & 510 \\
Krankenversicherung & 110 & 150 & 260 \\
Schaden-/Unfallversicherung & 9 & 599 & 608 \\
Gesamt & 271 & 1107 & 1378 \\
\hline
\end{tabular}

Tab. 7 Anzahl der Produktneuerungen in der Lebensversicherung im Zeitverlauf

\begin{tabular}{lll}
\hline & Anzahl & In Prozent \\
\hline 2006 & 63 & 11,8 \\
2007 & 48 & 9,0 \\
2008 & 49 & 9,2 \\
2009 & 68 & 12,7 \\
2010 & 39 & 7,3 \\
2011 & 33 & 6,2 \\
2012 & 33 & 6,2 \\
2013 & 38 & 7,1 \\
2014 & 45 & 8,4 \\
2015 & 46 & 8,6 \\
2016 & 36 & 6,7 \\
2017 & 37 & 6,9 \\
Gesamt & 535 & 100,0 \\
\hline
\end{tabular}

Die Betrachtung der Produkteinführungen im Zeitverlauf in Tab. 7 verdeutlicht dies: Jährlich sind mindestens 33 neue Lebensversicherungsprodukte auf den Markt gebracht worden, $43 \%$ davon sogar in den ersten vier Jahren. Die hohe Neuerungsquote zu Beginn des Untersuchungszeitraums lässt sich vor allem auf die Gesetzesänderungen 2005 zurückführen. Und das Jahr 2009 weist mit 68 Produktneuerungen am meisten auf. Hingegen lässt sich der Effekt des Lebensversicherungsreformgesetzes aus dem Jahr 2014 nicht so deutlich erkennen. Immerhin weisen die Jahre 2014 und 2015 jedoch um etwa $20 \%$ höhere Zahlen als die Jahre davor und danach aus.

Produktneuerungen in der Krankenversicherung Die Untersuchung für den Zeitraum von 1996 bis 2005 von Köhne/Kopp hat ergeben, dass die Krankenversicherung mit 135 von 650 Produktneuerungen (21\%) den geringsten Anteil an der Gesamtheit der Produktneuerungen ausgemacht hat. ${ }^{62}$ Im Zuge der empirischen Erhebung für den Zeitraum von 2006 bis 2017 konnten im Privatkundensegment 8 Produktinnovationen und 260 Produktmodifikationen im Krankenversicherungsbereich identifiziert werden (siehe Tab. 4). Betrachtet man wiederum die Jahresdurchschnitte

623 von 24 Innovationen und 132 von 626 Modifikationen, d.h. 135 von 650 Produktneuerungen; vgl. Köhne und Kopp (2007, S. 249). 
Tab. 8 Anzahl der Produktneuerungen in der Krankenversicherung nach Versicherungszweigen

\begin{tabular}{llll}
\hline & Innovationen & Modifikationen & Gesamt \\
\hline Betriebliche Krankenversicherung & 0 & 17 & 17 \\
Krankentagegeldversicherung & 0 & 3 & 3 \\
Krankenvollversicherung & 1 & 66 & 67 \\
Krankenzusatzversicherung & 3 & 61 & 64 \\
Pflegekostenversicherung & 0 & 23 & 23 \\
Pflegetagegeldversicherung & 0 & 27 & 27 \\
Reisekrankenversicherung & 0 & 23 & 23 \\
Service/Assistance & 4 & 3 & 7 \\
Tierkrankenversicherung & 0 & 2 & 2 \\
Zahnzusatzversicherung & 0 & 35 & 35 \\
Gesamt & 8 & 260 & 268 \\
\hline
\end{tabular}

für beide Untersuchungszeiträume, so kann mit 22,363 neu eingeführten Krankenversicherungsprodukten pro Jahr im Vergleich zum Zeitraum zwischen 1996 und 2005 mit 13,5 Neuerungen eine merkliche Steigerung auch in der Krankenversicherung konstatiert werden. Nach wie vor ist die Krankenversicherung jedoch im Vergleich die Versicherungszweiggruppe mit den wenigsten Produktneuerungen. Hier lässt sich also eine Trendfortsetzung beobachten, wenn auch insgesamt auf einem höheren Niveau.

Bezogen auf die verschiedenen Versicherungszweige konnte mit jeweils über 60 neuen Produkten die größte Neuerungsrate in der Krankenvoll- und Krankenzusatzversicherung festgestellt werden (Tab. 8). Gemeinsam mit der Zahnzusatz-, Pflegetagegeld-, Pflegekosten- und Reisekrankenversicherung wurden in diesen Zweigen $89 \%$ aller Neuerungen der Krankenversicherung eingeführt.

Die deutlich erhöhte Anzahl an Produktmodifikationen in der privaten Krankenversicherung kann auf diverse Umstrukturierungen und Anpassungen der gesetzlichen Krankenversicherung in den letzten Jahren zurückgeführt werden. Schon 2004 wurde mit dem GKV-Modernisierungsgesetz durch die Einführung von Zuzahlungsverpflichtungen zu zahlreichen Behandlungen und der Praxisgebühr von $10 €$ die Leistung spürbar beschränkt. Zugleich wurde den Krankenkassen die Möglichkeit geboten, Selbstbehaltstarife anzubieten. Dieses Gesetz war ein wesentlicher Impuls für die Entwicklung neuer Tarife in Form einer Umgestaltung bestehender Produkte und die Einführung von Zusatz- und Pflegeversicherungen. Diese Auswirkungen haben Köhne/Kopp zum Teil schon in ihrem Untersuchungszeitraum festgestellt. ${ }^{64}$ Sie wirken auch in das Jahr 2006 und somit in den Zeitraum dieser Untersuchung ein.

Weitergehend wurde die gesetzliche Krankenversicherung durch das GKV-Wettbewerbsstärkungsgesetz 2007 mit der Einführung von Gesundheitsfonds, einer weitergehenden Erlaubnis für Krankenkassen, Wahltarife mit einer gesetzlichen Bindefrist von drei Jahren anbieten zu dürfen, sowie der Regelung, dass chronisch Kranke

63 Der Wert ergibt sich wie folgt: 268 Produktneuerungen, dividiert durch 12 Jahre.

64 Vgl. Köhne und Kopp (2007, S. 244). 
ihre Zuzahlungsgrenze durch regelmäßige Vorsorgeuntersuchungen reduzieren können, reformiert. Die gesetzliche dreijährige Bindefrist für Wahltarife wurde durch das GKV-Finanzierungsgesetz ab dem 01.01.2011 auf ein Jahr abgesenkt. Zudem wurde seit 2015 mit dem GKV-Finanzstruktur- und Qualitätsentwicklungsgesetz ein individueller zusätzlicher Beitragssatz eingeführt, der allein den Versicherten belastet. ${ }^{65}$ Die Anpassungen in den privaten Pflege- und Zusatzversicherungen sind vor diesem Hintergrund auf die Leistungsreduzierungen der gesetzlichen Krankenkassen zurück zu führen. Für Versicherer war somit ein Anlass gegeben, neue Produkte in diesen Versicherungszweigen zu entwickeln und auf den Bedarf zu reagieren.

Insgesamt ist im Zeitraum von 2006 bis 2017 mit etwas über $42 \%$ ein beachtlicher Teil aller Produktmodifikationen in der Krankenversicherung auf politisch-rechtliche Neuerungen zurückzuführen (siehe Tab. 6).

Im Zusammenspiel mit den Erkenntnissen zur Lebensversicherung im vorherigen Abschnitt lässt sich als weiterer Befund somit Hypothese 4 bestätigen, dass in der Lebens- und in der Krankenversicherung ein Großteil der Produktneuerungen durch politisch-rechtliche Anstöße verursacht wird. Zusätzlich kann festgehalten werden, dass prozentual die Bedeutung in der Krankenversicherung noch höher ist, als in der Lebensversicherung.

Produktneuerungen in der Schaden-/Unfallversicherung Zwischen 2006 und 2017 sind in der Schaden-/Unfallversicherung im Privatkundensegment mit 46 die meisten Innovationen in den drei Versicherungszweiggruppen entstanden (siehe Tab. 4). Ähnlich verhält es sich auch im Bereich der Produktmodifikationen: In den untersuchten zwölf Jahren sind 608 modifizierte Produkte auf den Markt gebracht worden, im Spitzenjahr 2009 allein 77. Damit hat diese Versicherungszweiggruppe $58 \%$ aller Innovationen und $44 \%$ der Modifikationen ausgemacht. Das scheint die Erkenntnis von Köhne/Kopp zu bestätigen, dass die meisten Produktinnovationen auf Neuproduktideen im Sachgüterbereich zurückzuführen seien. ${ }^{66} \mathrm{Im}$ Vergleich zum Zeitraum von 1996 bis 2005 mit 238 Produktneuerungen (davon 17 Innovationen und 221 Modifikationen) sind das fast drei Mal so viele. Damit lässt sich über beide Beobachtungszeiträume der allgemeine Trend ableiten, dass im Schaden-/ Unfallbereich tendenziell mehr neue Produkte eingeführt werden als in der Lebensund Krankenversicherung.

Ebenfalls bestätigt sich die Beobachtung, dass in dieser Versicherungszweiggruppe kaum politisch-rechtlich angeregte Neuerungen festzustellen sind (siehe Tab. 6). Als einziger politisch-rechtlicher Anstoßpunkt konnte das Gesetz zur Angemessenheit der Vorstandsvergütung (VorstAG) von 2009 erfasst werden. Es schreibt vor, dass Vorstandsmitglieder und Entscheidungsträger trotz einer vorhandenen Unternehmens-D\&O-Versicherung mindestens zehn Prozent des Schadens bis zur Höhe des Eineinhalbfachen der festen jährlichen Vergütung des Betroffenen als Selbstbehalt tragen müssen. Als Reaktion darauf sind von Versicherungsunternehmen entsprechende D\&O-Selbstbehaltsversicherungen angeboten worden. Diese sind dem Segment der Privatkunden zugeordnet worden, weil sie meistens durch die Führungs-

65 Vgl. Bäcker (2017, S. 11-22).

66 Vgl. Köhne und Kopp (2007, S. 245). 
Tab. 9 Anzahl der Produktneuerungen in der Schaden-/Unfallversicherung nach Versicherungszweigen

\begin{tabular}{llll}
\hline & Innovationen & Modifikationen & Gesamt \\
\hline Unfallversicherung & 6 & 107 & 113 \\
Kfz-Versicherung & 7 & 103 & 110 \\
Hausratversicherung & 0 & 64 & 64 \\
Cyberversicherung & 0 & 4 & 4 \\
Wohngebäudeversicherung & 0 & 27 & 27 \\
Haftpflichtversicherung & 3 & 108 & 111 \\
Rechtsschutzversicherung & 4 & 58 & 62 \\
Bündelprodukte & 1 & 30 & 31 \\
Service-/Assistance-Produkte & 9 & 37 & 46 \\
Sonstige & 16 & 70 & 86 \\
Gesamt & 46 & 608 & 654 \\
\hline
\end{tabular}

kräfte als Privatpersonen abgeschlossen werden und das Interesse dieser Privatpersonen Gegenstand der Versicherung ist. Dennoch lassen sich im Untersuchungszeitraum lediglich fünf Produktneuerungen unmittelbar auf dieses Gesetz zurückführen. Es bleibt also dabei, dass politisch-rechtliche Änderungen für die Schaden-/ Unfallversicherung eher von geringer Bedeutung sind.

Weitere Befunde betreffen einzelne Versicherungszweige der Schaden-/Unfallversicherung. Die meisten Produktneuerungen sind in den Versicherungszweigen Unfall-, Kraftfahrt-, Haftpflicht-, Hausrat- und Rechtsschutzversicherung entstanden (Tab. 9). Vor allem in der Unfallversicherung ist ein Großteil der Modifikationen auf Veränderungen von Tarifbestandteilen zurückzuführen. So wurden häufig mehrere Produktvarianten mit verschiedenem Leistungsumfang angeboten. Dabei sind nur wenige Produkte erfasst worden, die eine bestimmte Zielgruppe ansprechen. Ein ähnliches Muster lässt sich in der Haftpflicht- und Hausratversicherung feststellen. Die meisten Modifikationen in diesen Zweigen sind also Produktvariationen im Sinne von Abschn. 3.2, die ohne ersichtliche politisch-rechtliche Anstoßpunkte oder gesellschaftliche Veränderungen eingeführt wurden.

Die große Anzahl der 110 Produktneuerungen in der Kraftfahrtversicherung ist auf die Investitionsoffensive in Forschung und Entwicklung der Automobilbranche, bedingt durch die veränderten Kundenerwartungen und die Digitalisierung, zurückzuführen. Die Versicherer sind bemüht, durch neue Produkte auf Trends wie Carsharing sowie die weiter fortschreitende Perfektionierung der Fahrerassistenzsysteme und Entwicklungen in Richtung des (teil)autonomen Fahrens zu reagieren. ${ }^{67}$ So sind im Beobachtungszeitraum zunehmend Telematik-Tarife für verschiedene Zielgruppen angeboten worden. ${ }^{68}$ Zugleich ist eine (wenn auch schwache) Reaktion der Versicherungsbranche auf das steigende Interesse an Old- und Youngtimerfahrzeugen innerhalb der Bevölkerung zu beobachten: ${ }^{69}$ Konkret sind vier Produktneuerungen für Besitzer von klassischen Fahrzeugen in den letzten sechs Jahren eingeführt

\footnotetext{
67 Vgl. Schmidt-Jochmann und Thiele (2015, S. 7 und 11 f.).

68 Für eine Darstellung zum Status quo im Jahr 2017 siehe Kraft und Hering (2017).

69 Vgl. auch Gelsdorf (2017, S. 48).
} 
worden. Zugleich sind aber auch vermehrt Versicherungsprodukte für Besitzer von Hybrid- und Elektrofahrzeugen entwickelt worden. Insgesamt kann daraus abgeleitet werden, dass die Versicherungsbranche in der Kraftfahrtversicherung sehr stark von der Digitalisierung und Innovationen im Sachgüterbereich der Automobilbranche beeinflusst wird.

Weitere Reaktionen auf die Digitalisierung in Form von neuen Versicherungsprodukten spiegeln sich unter anderem im Angebot von Cyber-, Drohnen-, Elektronikund Internet-Rechtsschutzversicherungen wider. Auch Service-/Assistanceprodukte werden verstärkt mit digitalen Komponenten angeboten: So ist beispielsweise im Jahr 2013 mithilfe des Telematik-Sicherheits-Service der Sparkassen DirektVersicherung AG eine Hilfsoption zur Verfügung gestellt worden, die durch eine im Fahrzeug verbaute Telematik-Box bei schweren Unfällen automatisch den nächst gelegenen Notarzt informieren und den Rettungswagen rufen kann. ${ }^{70}$

Hypothese 6, dass in der Schadenversicherung ein Großteil der Produktneuerungen auf die Digitalisierung und mithin technologische Entwicklungen zurückgeht, scheint sich somit zu bestätigen. Allerdings ist diesbezüglich eine weitergehende, konkretere Analyse der erfassten Versicherungsprodukte notwendig. ${ }^{71}$

Bei der Betrachtung der Produktneuerungen in der Schaden-/Unfallversicherung fällt besonders auf, dass 46 Produkte - das entspricht $7 \%$ aller Produktneuerungen in dieser Versicherungszweiggruppe - Service- bzw. Assistanceprodukte sind, die darauf ausgelegt sind, dem Versicherungsnehmer eine Hilfe oder einen Service auf einem bestimmten Gebiet zu bieten. Diese werden zum Teil unentgeltlich als Zusatz zu bestehenden Versicherungsverträgen oder aber als eigenständiges Produkt angeboten. Während zwischen 1996 und 2005 nur 16 Produkte dieser Art erfasst worden sind, sind es nunmehr fast drei Mal so viele. Dennoch muss Hypothese 7, dass in der Schadenversicherung ein Großteil der Produktneuerungen auf Service- und Assistanceleistungen zurückgeht, damit verworfen werden. $7 \%$ sind zwar bedeutend, und die Bedeutung nimmt zu, aber es handelt sich bei weitem nicht um den Großteil der Produktneuerungen.

Ergänzend anzumerken ist, dass diese Entwicklung nicht nur die Schaden-/ Unfallversicherung betrifft, denn auch in anderen Zweigen sind solche Produkte zu verzeichnen: Über alle Kundensegmente und Versicherungszweiggruppen hinweg sind insgesamt 76 neue Service- bzw. Assistanceprodukte erfasst worden.

\subsubsection{Unternehmensbezogene Befunde in Bezug auf Produktneuerungen}

Weitergehend lässt sich erkennen, wie viele Produktneuerungen von den Versicherungsunternehmen und -gruppen innerhalb der untersuchten zwölf Jahre auf dem Versicherungsmarkt angeboten worden sind (Tab. 10). Es fällt auf, dass $62 \%$ der Unternehmen in diesem Zeitraum nur 1-4 Produktneuerungen lanciert haben; das er-

\footnotetext{
70 Vgl. o. V. (2013, S. 22).

71 Das liegt darin begründet, dass nicht bei allen Produkten die Einflussintensität der Digitalisierung erkannt werden konnte, sei es bezogen auf das neu gedeckte Risiko oder auf das Versicherungsprodukt an sich.
} 
Tab. 10 Anzahl an Versicherungsunternehmen mit jeweiliger Anzahl ihrer Produktneuerungen über alle Kundensegmente

\begin{tabular}{lll}
\hline $\begin{array}{l}\text { Anzahl Produkt- } \\
\text { neuerungen }\end{array}$ & $\begin{array}{l}\text { Anzahl Unter- } \\
\text { nehmen }\end{array}$ & $\begin{array}{l}\text { Anteil an Unterneh- } \\
\text { men in \% }\end{array}$ \\
\hline $1-4$ & 176 & 62 \\
$5-20$ & 88 & 31 \\
Über 20 & 19 & 7 \\
Gesamt & 283 & 100 \\
\hline
\end{tabular}

Tab. 11 Versicherungsunternehmen im Privatkundensegment mit den meisten Produktneuerungen (PN)

\begin{tabular}{ll}
\hline Name des Versicherungsunternehmens & Anzahl der PN \\
\hline Basler Sachversicherungs-AG & 32 \\
Münchener Verein Krankenversicherung a. G. & 30 \\
Stuttgarter Lebensversicherung a. G. & 30 \\
HDI Lebensversicherung AG & 29 \\
Zurich Life Assurance plc & 27 \\
Allianz Versicherungs-AG & 27 \\
Gothaer Lebensversicherung AG & 25 \\
Janitos Versicherung AG & 24 \\
Nürnberger Allgemeine Versicherungs-AG & 23 \\
ALTE LEIPZIGER Lebensversicherung auf Gegenseitigkeit & 21 \\
uniVersa Allgemeine Versicherung AG & 21 \\
ARAG Allgemeine Versicherungs-AG & 21 \\
HDI Versicherung AG & 21 \\
Swiss Life Pensionskasse AG & 21 \\
\hline
\end{tabular}

scheint wenig. Nur 19 Unternehmen bzw. $7 \%$ der Unternehmen haben über 20 Neuerungen hervorgebracht.

Im Privatkundensegment haben die 14 Unternehmen mit der höchsten Neuerungsaktivität in den zwölf Jahren zwischen 21 und 32 Produkte eingeführt (Tab. 11). Dabei ist ersichtlich, dass nicht immer die Höhe der Beitragseinnahmen und die Größe des Versicherungsunternehmens bestimmen, wie viele neue Produkte entwickelt und eingeführt werden. So hat im Bereich der Schaden-/Unfallversicherung die vergleichsweise kleine Basler Sachversicherungs-AG die meisten Produktneuerungen hervorgebracht, während beispielsweise die Zurich Versicherung in den letzten zwölf Jahren lediglich 9 neue Produkte eingeführt hat.

\section{Fazit}

In der vorliegenden Untersuchung sind die Anzahl an Produktinnovationen und Produktmodifikationen in der Versicherungswirtschaft zwischen 2006 und 2017 und deren Entwicklung im Zeitverlauf mittels einer umfassenden Aufarbeitung ermittelt sowie vor dem Hintergrund der Ergebnisse einer gleichartigen Analyse für die Jahre 1996 bis 2005 reflektiert worden. Als ersten wesentlichen Befund hat sie aufgezeigt, dass die Anzahl der Produktneuerungen zwischen 2006 und 2017 im 
Gesamtmarkt und gerade im Privatkundenbereich im Vergleich zum Zeitraum von 1996 bis 2005 deutlich zugenommen hat: In jüngster Zeit sind im Privatkundenbereich durchschnittlich jährlich 121 Produkte auf den Versicherungsmarkt gebracht worden, früher demgegenüber nur durchschnittlich 65 Neuerungen pro Jahr. Indessen hat sich der Trend, dass es auf dem Versicherungsmarkt erheblich weniger Innovationen als Modifikationen gibt, nachweisbar fortgesetzt: Nach wie vor gibt es mit fast $95 \%$ der Produktneuerungen erheblich mehr Produktmodifikationen als Produktinnovationen, wenngleich sich die Relation von Innovation zu Modifikation zugunsten der Innovation leicht verändert hat.

Mit Bezug auf die Versicherungszweiggruppen im Privatkundensegment kann sowohl für die Lebens-, als auch für die Krankenversicherung (erneut) festgehalten werden, dass ein Großteil der Produktneuerungen durch politisch-rechtliche Anstöße verursacht wird. Die empirische Untersuchung hat zudem vor Augen geführt, dass klassische Lebensversicherungsprodukte mit Garantiezins in zunehmendem Ausmaß von Alternativen ohne Garantien wie vor allem fondsgebundenen Lebensversicherungen abgelöst werden. ${ }^{72}$ Im Versicherungszweig Schaden-/Unfallversicherung finden bei Weitem die meisten Produktmodifikationen, aber auch Produktinnovationen statt, welche oft Technologie- oder Risiko-induziert sind, und der Anteil an Service-orientierten Produkten nimmt zu. Politisch-rechtliche Impulse haben hier eine geringe Bedeutung.

Schließlich hat sich bestätigt, dass es keinen deutlich zu erkennenden Trend im 12-jährigen Beobachtungszeitraum gibt. Zwar gibt es Schwankungen, die meist aus politisch-rechtlichen Neuregelungen resultieren, aber ansonsten bleibt die Anzahl an Produktneuerungen recht stabil.

Die im Vergleich zur damaligen Untersuchung festgestellte Zunahme von Produktneuerungen geht offenbar nicht auf die Deregulierung von 1994 zurück, sondern auf technologische und Kapitalmarktentwicklungen sowie zunehmende politischrechtliche Impulse, die im Zeitraum von 2006 bis 2017 fast zu einer Verdopplung der Innovationen und auch der Modifikationen geführt haben. Die vergleichsweise höhere Anzahl an Innovationen in der Lebensversicherung dürfte auf die nachhaltige Veränderung der Kapitalmarktsituation nach der Finanzkrise von 2007 und die damals bevorstehende Einführung von Solvency II im Jahr $2016^{73}$ zurückzuführen sein. Schließlich sind eine deutliche Zunahme der Zielgruppenorientierung (von knapp 10 auf $28 \%$ der Produktneuerungen) sowie ein zunehmender Anteil an Serviceprodukten beobachtet worden - Beides ist Ausdruck zunehmender Kundenorientierung, die als Signal zunehmenden Wettbewerbs interpretiert wird.

Mithin hat das Produktneuerungsgebaren der Versicherer im deutschen Privatkundenmarkt in dem Zeitraum von 2006 bis 2017 im Vergleich zu den zehn Jahren davor offensichtlich zugenommen, und das in allen Versicherungszweiggruppen. Die Produktpolitik wird zunehmend als wichtiges Marketinginstrument erkannt und genutzt, wenngleich es immer noch viele Unternehmen gibt, die nur wenige Produktneuerungen auf den Markt bringen.

\footnotetext{
72 Damit bestätigt sie die Erkenntnisse einer qualitativen Studie von Bierth u. a. aus dem Jahr 2018 (vgl. Bierth et al. 2018, S. 134).

73 Dieser Befund deckt sich mit einer Untersuchung von Klotzki (vgl. Klotzki 2018, S. 158f.).
} 
Unter methodischen Gesichtspunkten lässt sich festhalten, dass die vorliegende Untersuchung auf einem Datensatz von 1750 identifizierten Produktneuerungen über einen Zeitraum von 12 Jahren basiert. Sie hat einige interessante Aussagen zur Gesamtentwicklung von Produktneuerungen generiert und dabei sowohl eine Differenzierung nach Innovationen und Modifikationen sowie nach Lebens-, Krankenund Schaden-/Unfallversicherung, als auch einen Vergleich zu einer früheren, zeitlich vorgelagerten Studie ermöglicht. Aufgrund der deskriptiv angelegten Erhebung lässt sie jedoch keine Aussagen zu Ursache-Wirkungs-Zusammenhängen zu. Ihre vor allem quantitative Ausrichtung hat zudem dazu geführt, dass nur wenige Aussagen zu den Inhalten der Produktneuerungen und diesbezüglichen Trends extrahiert werden konnten. Hier wäre eine weitere, qualitativ ausgerichtete Studie erkenntnisfördernd. Eine weitere Einschränkung ergibt sich im Hinblick auf die Erfassung des Digitalisierungseinflusses auf Produktneuerungen: Zwar konnte dieser Einfluss mit Bezug auf die unmittelbare Wirkung auf den entsprechenden Cyberrisiko-Versicherungsschutz ermittelt werden. Aber der mittelbare Einfluss der Digitalisierung auf die übrigen Produktneuerungen war - bedingt durch die herangezogenen Quellen nur ansatzweise zu erkennen. Das gilt insbesondere für die Analyse der Bedeutung von InsurTechs im Zuge der Entwicklung von Produktneuerungen; hierfür wäre eine umfassende Sichtung der entsprechenden Internet-Homepages deutlich erkenntnisreicher. Auch das bleibt einer gesonderten Untersuchung vorbehalten.

Im Hinblick auf künftige Entwicklungen wird es also vor dem Hintergrund der aktuell zahlreich in den Markt eintretenden InsurTechs spannend sein zu beobachten, inwiefern diese sich überhaupt am Markt etablieren und dadurch die künftige Produktentwicklung beeinflussen. Das Gleiche gilt für die sogenannten Ökosysteme, an denen sich verschiedene Unternehmen beteiligen und durch kooperative Vernetzung am Kundennutzen orientierte Produkt- und Dienstleistungsbündel kreieren. Die Vernetzung basiert überdies oft auf digitalen Prozessen und unterstützt die umfassende Sammlung von Kundendaten (Big Data). Insofern fließen hier verschiedene Entwicklungen zusammen, und es gilt zu beobachten, welche Einflüsse das auf die künftige Produktentwicklung haben wird: ${ }^{74}$ Beispielsweise wird es interessant sein $\mathrm{zu}$ verstehen, ob und wie die weitere Telematikentwicklung die Kfz-Versicherung nicht nur preisbezogen, sondern auch hinsichtlich der erweiterten Produktleistungen verändern wird. Ebenso ist der Einfluss von digitalen Datenmessungen im Krankenversicherungsbereich auf die Produktleistungen zu ermitteln, z. B. durch die Nutzung von Fitness-Trackern ${ }^{75}$, und in der Hausrat- und Wohngebäudeversicherung könnte die Weiterentwicklung von Smart home-Technologien die Versicherungsprodukte verändern. ${ }^{76}$ Aus der Ökosystem-Logik heraus sind künftig ebenso Wettbewerber aus anderen Branchen als Treiber (gänzlich) neuer Produktkonzepte denkbar; erste ,Vorboten' sind beispielsweise der Autobauer Tesla, der seinen Kunden beim Autokauf den Kfz-Versicherungsschutz inklusive anbieten möchte, oder der Versandhändler Amazon, der - wenngleich derzeit noch nur in vereinzelten Ländern

\footnotetext{
74 Vgl. Eling und Lehmann (2018, S. 375-378).

75 Eine aktuelle Untersuchung befasst sich mit der möglichen Akzeptanz dieser Technologien auf Kundenseite (vgl. Wiegard et al. 2019).

76 Für eine Darstellung zum Status quo im Jahr 2018 siehe Barb et al. (2018).
} 
und mit ausgewählten Produkten - in zunehmendem Ausmaß mit Versicherungsprodukten experimentiert. Eine weitere Triebfeder künftiger Produktneuerungen ist die demografische Entwicklung: In der Lebensversicherung wird dadurch bedingt die innovative Ausgestaltung der Rentenphase in Zukunft an Bedeutung gewinnen (müssen).

Funding Open Access funding provided by Projekt DEAL.

Open Access Dieser Artikel wird unter der Creative Commons Namensnennung 4.0 International Lizenz veröffentlicht, welche die Nutzung, Vervielfältigung, Bearbeitung, Verbreitung und Wiedergabe in jeglichem Medium und Format erlaubt, sofern Sie den/die ursprünglichen Autor(en) und die Quelle ordnungsgemäß nennen, einen Link zur Creative Commons Lizenz beifügen und angeben, ob Änderungen vorgenommen wurden.

Die in diesem Artikel enthaltenen Bilder und sonstiges Drittmaterial unterliegen ebenfalls der genannten Creative Commons Lizenz, sofern sich aus der Abbildungslegende nichts anderes ergibt. Sofern das betreffende Material nicht unter der genannten Creative Commons Lizenz steht und die betreffende Handlung nicht nach gesetzlichen Vorschriften erlaubt ist, ist für die oben aufgeführten Weiterverwendungen des Materials die Einwilligung des jeweiligen Rechteinhabers einzuholen.

Weitere Details zur Lizenz entnehmen Sie bitte der Lizenzinformation auf http://creativecommons.org/ licenses/by/4.0/deed.de.

\section{Literatur}

Bäcker, G.: Chronologie gesetzlicher Neuregelungen - Krankenversicherung \& Gesundheitswesen 1998-2016 (2017)

BaFin: Big Data trifft auf künstliche Intelligenz. Herausforderungen und Implikationen für Aufsicht und Regulierung von Finanzdienstleistungen (2018)

Barb, C., Klein, K., Kraft, M.: Auswirkungen von Smart-Home-Technologien auf die verbundene Hausrat- und Wohngebäudeversicherung - Markt, Ausgestaltungsmerkmale und Preisbereitschaft. Z. Ges. Versicherungswiss. 107(5), 495-516 (2018)

Bierth, C., Friedrich, K., Linderkamp, T., Lohse, U., Schröder, M.: Zukunft der Versicherung - Versicherung der Zukunft. Z. Ges. Versicherungswiss. 107(2), 127-141 (2018)

Brauns, B.: Altersvorsorge - Die Lebensverunsicherer (2016). https://www.zeit.de/wirtschaft/geldanlage/ 2016-04/lebensversicherung-altersvorsorge-altersarmut-pleite-geldanlage-renditeverbraucherschutz. Zugegriffen: 6. Apr. 2019

Brühl, V., Walz, U.: Das anhaltende Niedrigzinsumfeld in Deutschland. CFS Working Paper Series, Bd. 506. (2015)

Bundesministerium für Arbeit und Soziales: Forschungsbericht 475 - Arbeitgeber- und Trägerbefragung zur Verbreitung der betrieblichen Altersversorgung (BAV 2015), Endbericht (2016)

De Ridder, W.P., Ruß, J., Seyboth, A.: Flexible Renten. In: Schiereck, D., Haupt, T., Neuenfeldt, O. (Hrsg.) Ruhestandsplanung - neuer Beratungsansatz für die Zielgruppe 50plus, S. 69-87. Gabler, Wiesbaden (2015)

Deutsche Aktuarvereinigung e. V.: Lebensversicherungsprodukte unter Solvency II: Der Beginn einer neuen Ära? Aktuar Aktuell 38(7), 12-13 (2017)

Eling, M., Lehmann, M.: The impact of digitalization on the insurance value chain and the insurability of risks. Geneva Pap Risk Insur Issues Pract 43(3), 359-396 (2018)

ERGO Lebensversicherung AG: Geschäftsbericht 2018 (2019)

Farny, D.: Die Gestaltung von Versicherungsprodukten im Marketing von Versicherungsunternehmen. Z. Ges. Versicherungswiss. 84(1/2), 79-102 (1995)

Farny, D.: Versicherungsbetriebslehre, 5. Aufl. Verlag Versicherungswirtschaft, Karlsruhe (2011)

FRI:DAY: Monatlich zahlbar, Monatlich kündbar, Die flexible FRIDAY Original Autoversicherung (2019). https://www.friday.de/kfz-versicherung/friday-original. Zugegriffen: 6. Apr. 2019

Gaedeke, O.: Klartext kämpft gegen Low Interest - Besonderheiten der Marktforschung in der Assekuranz. Gastbeitrag. In: Köhne, T. (Hrsg.) Versicherungsmarketing. Marketing und Vertrieb im Versicherungsunternehmen in Theorie und Praxis, S. 88-93. Verlag Versicherungswirtschaft, Karlsruhe (2016) 
Gal, J.: Rechtliche Rahmenbedingungen eines Versicherungsmarketing. In: Reich, M., Zerres, C. (Hrsg.) Handbuch Versicherungsmarketing, 2. Aufl., S. 97-119. Springer, Heidelberg, Berlin, New York (2019)

Gelsdorf, R.: Die rasante Entwicklung am Kfz-Markt erfordert neue Versicherungslösungen. AssCompact 9, 48 (2017)

Generali Deutschland AG: Aktuelle Informationen für Kunden der Generali Lebensversicherung (seit 7. Oktober Proxalto Lebensversicherung) (2019). https://www.generali.de/ueber-generali/ueber-uns/ generali-versicherungen/kundeninformation/. Zugegriffen: 1. Nov. 2019

Getsafe: Die Getsafe Haftpflicht (2019). https://www.hellogetsafe.com/de-de/leistungsdetails/haftpflicht. Zugegriffen: 6. Apr. 2019

Hepster: Die beste Reiseunfallversicherung für Dich! (2019). https://buchung.hepster.com/reiseunfallver sicherung. Zugegriffen: 6. Apr. 2019

Klotzki, U.: New life insurance products and product complexity in Germany. Z. Ges. Versicherungswiss. 107(2), 143-161 (2018)

Köhne, T.: Versicherungsmarketing. Marketing und Vertrieb im Versicherungsunternehmen in Theorie und Praxis. Verlag Versicherungswirtschaft, Karlsruhe (2016)

Köhne, T., Kopp, F.: Produktinnovationen und Produktmodifikationen in der Versicherungswirtschaft: Rolle und Bedeutung nach der Deregulierung. Z. Ges. Versicherungswiss. 96(2), 227-259 (2007)

Kotalakidis, N., Mueller, F., Naujoks, H.: Digitalisierung der Versicherungswirtschaft: Die 18-MilliardenChance (2016)

KPMG: Zukunft der Lebensversicherung - Insurance Thinking Ahead (2018)

Kraft, M., Hering, J.: Potenziale von Telematik-Tarifen in der Kfz-Versicherung in Deutschland, Theoretische Überlegungen und empirische Ergebnisse zur Akzeptanz. Z. Ges. Versicherungswiss. 106(5), 503-524 (2017)

Lach, H.: Vertikales Marketing von Versicherungsunternehmen, Marketingkonzepte für Versicherungsunternehmen mit Ausschließlichkeits-, Makler- und Strukturvertrieb. Duncker \& Humblot, Berlin (1995)

Lohse, U., Will, A.: Rahmenbedingungen und strategische Herausforderungen für die Versicherungsbranche. In: Reich, M., Zerres, C. (Hrsg.) Handbuch Versicherungsmarketing, 2. Aufl., S. 3-14. Springer, Heidelberg, Berlin, New York (2019)

MAXCARE: Für Ihre Zähne nur das Beste: MAXCARE Zahnzusatzversicherung (2019). https://www. maxcare.de. Zugegriffen: 6. Apr. 2019

MSR Insights, W\&W Digital GmbH, etventure GmbH: Studie „Versicherungen für Digital Natives“: Versicherer verlieren die Zielgruppe der Digital Natives an Insurtechs, Pressemitteilung vom 2. Nov. 2016 (2016)

Müller-Reichart, M., Geist, P.: Service als existenzsicherndes Geschäftsmodellattribut der Versicherungswirtschaft, Das Assistance-Barometer 2015. Z. Versicherungswes. 66(8), 254-259 (2015)

Müller-Reichart, M., Bersch, K.L., Haisch, D.: Assistance als Schlüssel zu digitalen Ökosystemen der Versicherungsnehmer. AssCompact 5, 34-37 (2019)

Oliver Wyman, Policen Direkt: Zukunft von InsurTech in Deutschland. Der InsurTech Radar 2017 (2017)

o. V.: Sparkassen DirektVersicherung startet mit Telematik-Sicherheits-Service. AssCompact 12, 22 (2013)

Reuß, A., Ruß, J., Wieland, J.: Participating life insurance products with alternative guarantees: Reconciling policyholders' and insurers' interests. Risks 4(11), 1-18 (2016)

Ruß, J., Schiller, J., Seyboth, A.: Regulierung von Provisionen. Ziele, Risiken und Nebenwirkungen provisionsbegrenzender Regulierung in der Lebensversicherung in Deutschland (2018)

Schmeiser, H., Eling, M., Gatzert, N., Schuckmann, S., Toplek, D.: Volkswirtschaftliche Implikationen des Swiss Solvency Tests. I.VW Schriftenreihe, Bd. 48. St. Gallen (2006)

Schmidt-Jochmann, C., Thiele, J.: Geschäftsmodell der KFZ-Versicherung im Umbruch. In: Roland Berger GmbH (Hrsg.) Studie der Roland Berger Strategy Consultants, Bd. 9. (2015)

Surminski, M.: Axa im Run-off-Modus. Z. Versicherungswes. 69(17), 503 (2018)

Swiss Re: Produktinnovation in der Nichtlebensversicherung - Von kleinen und grossen Innovationen. In: sigma, Bd. 4. (2011)

Theis, A., Wiener, K.: Anbieterlandschaft am Versicherungsmarkt: Ein Ausblick. In: Gesamtverband der deutschen Versicherungswirtschaft (Hrsg.) Volkswirtschaftliche Themen und Analysen, Bd. 8. (2018)

vbw Vereinigung der Bayerischen Wirtschaft e. V.: Studie Digitalisierung in der Versicherungswirtschaft (2017)

Wagner, F. (Hrsg.): Gabler Versicherungslexikon, 2. Aufl. Gabler, Wiesbaden (2017) 
Wichert, B.: Was der Vertrieb über die Silver Ager wissen sollte (2016). http://www.versicherungsjournal. de/vertrieb-und-marketing/was-der-vertrieb-ueber-die-silver-ager-wissen-sollte-125524.php. Zugegriffen: 6. Apr. 2019

Wiegard, R., Guhr, N., Krylow, S., Breitner, M.H.: Analysis of wearable technologies' usage for payas-you-live tariffs: Recommendations for insurance companies. Z. Ges. Versicherungswiss. 108(1), 63-88 (2019)

Wiener, K., Theis, A.: InsurTech(s): Zwischen Konkurrenz und Partnerschaft. In: Gesamtverband der deutschen Versicherungswirtschaft (Hrsg.) Makro und Märkte kompakt, Bd. 9 (2017) 